\title{
Stable intersections of Cantor sets and homoclinic bifurcations
}

\author{
by
}

\author{
Carlos Gustavo T. de A. MOREIRA
}

IMPA, Edificio Lelio Garra, Estrada Dona

Castorina 110, Jardim Botanico, Rio de Janeiro, CEP 22460 Brasil.

\begin{abstract}
We study intersections of dynamically defined Cantor sets and consequences to dynamical systems. The concept of stable intersection of two dynamically defined Cantor sets is introduced. We prove that if the stable and unstable Cantor sets associated to a homoclinic bifurcation have a stable intersection, then there are open sets in the parameter line with positive density at the initial bifurcating value, for which the corresponding diffeomorphisms are not hyperbolic. We present conditions more general than the ones previously known that assure stable intersections. We also present conditions for hyperbolicity to be of positive density at homoclinic bifurcations. This allow us to provide persistent one-parameter families of homoclinic bifurcations that present both hyperbolicity and homoclinic tangencies with positive density at the initial bifurcating value in the parameter line.
\end{abstract}

Résumé. - Nous étudions des intersections d'ensembles de Cantor et ses conséquences sur les systèmes dynamiques. Le concept d'intersection stable de deux ensembles de Cantor dynamiquement définis est ici introduit. Nous prouvons que si les ensembles de Cantor stable et instable associés à une bifurcation homoclinique ont une intersection stable, alors il y a des ensembles ouverts dans la ligne de paramètres avec densité positive dans la valeur initiale de bifurcation pour lesquels les difféomorphismes ne sont pas hyperboliques.

Nous présentons des conditions qui assurent des intersections stables, plus générales que les conditions préalablement connues. Nous présentons aussi

Classifications A.M.S.: $58 \mathrm{~F} 14,58 \mathrm{~F} 15$.

Annales de l'Institut Henri Poincaré - Analyse non linéaire - 0294-1449

Vol. 13/96/06/\$ 7.00/

(C) 1996 L'Association Publications de l'Institut Henri Poincaré. Published by Elsevier B.V. All rights reserved 
des conditions pour que l'hyperbolicité ait densité positive aux bifurcations homocliniques. Cela nous permet de présenter des exemples persistents de familles de bifurcations homocliniques à un paramètre qui présentent en même temps de l'hyperbolicité et des tangences homocliniques avec densité positive dans la valeur initiale de bifurcation dans la ligne de paramètre.

\section{INTRODUCTION}

The objective of this work is to study intersections of Cantor sets on the real line which are typical of the Cantor sets that appear in dynamical systems: the dynamically defined Cantor sets. Indeed, they appear naturally in the study of hyperbolic sets, like Smale's horseshoe, which is the product of two Cantor sets of this kind. They also appear in different mathematical contexts. For instance, for any $m \in \mathbf{N}$ bigger than one, the set of numbers in the interval $[0,1]$ whose continued fractions have all its coefficients less than or equal to $m$ is a Cantor set of this kind. Roughly speaking, dynamically defined or regular Cantor sets are Cantor sets that are defined by expanding functions (see the precise definition in Section I).

The original motivation for this work was a conjecture of J. Palis, according to which generically the arithmetic difference $K_{1}-K_{2}=\{x-y \mid$ $\left.x \in K_{1}, y \in K_{2}\right\}$ of two dynamically defined Cantor sets either has measure zero or else contains intervals. The study of measure-theoretical and topological properties of arithmetic differences of subsets of the realline is particularly delicate for Cantor sets. For other classes of subsets of $\mathbf{R}$, the following results about arithmetic differences are known, and their proofs are quite simple:

- $A$ has measure zero and $B$ is countable $\Rightarrow A-B$ has measure zero;

- $A$ is of first category and $B$ is countable $\Rightarrow A-B$ is of first category;

- $A$ and $B$ have positive measure $\Rightarrow A-B$ contains an interval;

- $A$ and $B$ are residual $\Rightarrow A-B=\mathbf{R}$.

On the other hand, concerning dynamically defined Cantor sets, several relevant results have been obtained, such as:

- If $H D\left(K_{1}\right)+H D\left(K_{2}\right)<1$ (where $H D(K)$ denotes the Hausdorff dimension of $K$ ) then $K_{1}-K_{2}$ has measure zero;

- If $\tau\left(K_{1}\right) \cdot \tau\left(K_{2}\right)>1$ then $K_{1}-K_{2}$ contains an interval.

Here $\tau(K)$ denotes the thickness of $K$ (see Section II). 
These results can be easily proved, but involve subtle concepts with relevant applications in dynamics. The second is proved in Section II in a somewhat more general form, and the proof of the first one is sketched in Section I.1.

The concept of thickness was used by Newhouse [N1] to exibit open sets of diffeomorphisms with persistent homoclinic tangencies, therefore without hyperbolicity. It is possible [N2] to prove that in such an open set there is a residual set of diffeomorphisms which present infinitely many coexisting sinks. In [N3], it is proved that under generic hypotheses every family of surface diffeomorphisms that unfold a homoclinic tangency goes throught such an open set. A new and perhaps clearer proof is in [PT2].

In this work, we introduce yet another concept about Cantor sets related to dynamical systems: we say that two dynamically defined Cantor sets $K_{1}$ and $K_{2}$ have stable intersection if for any pair of dynamically defined Cantor sets $\left(\widetilde{K}_{1}, \widetilde{K}_{2}\right)$, near $\left(K_{1}, K_{2}\right)$ (in a certain topology), we have that $\widetilde{K}_{1} \cap \widetilde{K}_{2} \neq \emptyset$. The study of stable intersections of Cantor sets has close relations with the study of arithmetic differences of Cantor sets. For instance, if $\left(K_{1}, K_{2}\right)$ has stable intersection then $K_{1}-K_{2}$ contains an interval. Theorem I-1 presents a kind of converse to this fact. On the other hand, it is easy to show that having stable intersection for two regular Cantor sets is more general than having the product of their thicknesses bigger than one.

This work is essentially dedicated to the study of stable intersections of dynamically defined Cantor sets and its consequences about the study of homoclinic bifurcations.

In Section I, we initially present some basic concepts and explain the relationship between homoclinic bifurcations and intersections of Cantor sets. In simple terms, we consider a one-parameter family $\left(\varphi_{\mu}\right), \mu \in \mathbf{R}$, of diffeomorphisms which unfold a homoclinic tangency at $\mu=0$, associated to a saddle point $p$ belonging to a non trivial basic set $\Lambda$, i.e., to a hyperbolic maximal invariant set in which the periodic points are dense. We can associate to $\Lambda_{\mu}$, the continuation of $\Lambda_{0}=\Lambda$, two dynamically defined Cantor sets $\left(K_{1}\right)_{\mu}$ and $\left(K_{2}\right)_{\mu}$, the "factors" of $\Lambda_{\mu}$, such that, if $\left(K_{1}\right)_{\mu} \cap\left(K_{2}\right)_{\mu} \neq \emptyset$, then the stable and unstable foliations of $\Lambda_{\mu}$ have a point of tangency. In turn, such a tangency can be approximated by homoclinic tangencies associated to the point $p_{\mu}$ (the continuation of the saddle point $p_{0}=p$ ). In particular, if $\left(K_{1}\right)_{\mu} \cap\left(K_{2}\right)_{\mu} \neq \emptyset$, the diffeomorphism $\varphi_{\mu}$ is not hyperbolic.

We prove (Theorem I.2), that if $\left(K_{1}\right)_{0}$ has a stable intersection with $\left(K_{2}\right)_{0}+t$ for some value of $t \in \mathbf{R}$, then the set $\left\{\mu>0 \mid\left(K_{1}\right)_{\mu}\right.$ intersects 
$\left(K_{2}\right)_{\mu}$ stably $\}$ has positive density at $\mu=0$. We also introduce the concept of extremal stable intersection in Section I, and prove that if $\left(\left(K_{1}\right)_{0},\left(K_{2}\right)_{0}\right)$ has extremal stable intersection, then $\left(K_{1}\right)_{\mu} \cap\left(K_{2}\right)_{\mu} \neq \emptyset$ for every $\mu>0$ small.

In Section II, we introduce the concepts of thickness and lateral thicknesses, that allows us, among other things, to give examples of pairs of Cantor sets $\left(K_{1}, K_{2}\right)$ which have stable or extremal stable intersections and yet the product of their thicknesses may be smaller than one. Indeed, these conditions we use in these examples concern instead the Hausdorff dimensions $H D\left(K_{1}\right)$ and $H D\left(K_{2}\right)$ of $K_{1}$ and $K_{2}$ : We can give such examples for Cantor sets $K_{1}$ and $K_{2}$, with their Hausdorff dimensions having any value between zero and one and their sum being bigger than one.

In Section III, we discuss some examples about arithmetic differences and stable intersections.

In Section IV, we discuss more general criteria for stable and extremal stable intersections and provide examples of their applications.

In Section V, we exhibit conditions on the Cantor sets $\left(K_{1}\right)_{0}$ and $\left(K_{2}\right)_{0}$ which imply that the set $\left\{\mu>0 \mid \varphi_{\mu}\right.$ is persistently hyperbolic $\}$ has positive density at $\mu=0$. Besides, we give examples of open sets of one-parameter families of diffeomorphisms which unfold homoclinic tangencies, such that the phenomena of hyperbolicity and persistent tangencies between the stable and unstable foliations have both uniformly positive densities at $\mu=0$.

We also introduce the concept of extremal stable intersection, which is stronger than the concept of stable intersection, and have relevant application in dynamics: we say that $\left(K_{1}, K_{2}\right)$ have extremal stable intersection if the right extreme of $K$ coincides with the left one of $K_{2}$, and for any pair of two dynamically defined Cantor sets $\left(\tilde{K}_{1}, \tilde{K}_{2}\right)$ near $\left(K_{1}, K_{2}\right)$ which support intervals intersects we have $\tilde{K}_{1} \cap \tilde{K}_{2} \neq \emptyset$. It is easy to prove that, in the case of homoclinic bifurcations as above, if $\left(\left(K_{2}\right)_{0},\left(K_{2}\right)_{0}\right)$ has an extremal stable intersection, then $\left(K_{1}\right)_{\mu} \cap\left(K_{2}\right)_{\mu} \neq \emptyset$ for every $\mu>0$ small. We shall study here conditions that imply extremal stable intersections.

In Section $\mathrm{V}$ we shall prove a theorem that, under certain hypothesis about the Cantor sets $\left(K_{1}\right)_{0}$ and $\left(K_{2}\right)_{0}$ assures that the set $\left\{\mu>0 \mid \varphi_{\mu}\right.$ is persistently hyperbolic $\}$ has positive density at $\mu=0$. Besides, we give an example of an open set of 1-parameter families of diffeomorphisms which unfold homoclinic tangencies such that the phenomena of hyperbolicity and persistent tangencies between the stable and unstable foliations have both uniformly positive densities at $\mu=0$. 
This work is divided as follows:

Section I: Cantor sets and homoclinic bifurcations

Section II: Thickness and lateral thickness

Section III: Examples about arithmetic differences and stable intersections

Section IV: Criteria for stable and extremal stable intersections and generalized thickness test

Section V: Intersections of Cantor sets and hyperbolicity.

\section{CANTOR SETS AND HOMOCLINIC BIFURCATIONS}

\section{I.0. Basic definitions}

Given a diffeomorphism $\varphi: M \stackrel{C^{k}}{\rightarrow} M$ of a surface and $p$ a fixed point of $\varphi$, we define:

$$
\begin{aligned}
& W^{s}(p):=\left\{x \in M \mid \lim _{n \rightarrow \infty} \varphi^{n}(x)=p\right\} \quad \text { (the stable manifold of } p \text { ) } \\
& W^{u}(p):=\left\{x \in M \mid \lim _{n \rightarrow-\infty} \varphi^{n}(x)=p\right\} \quad \text { (the unstable manifold of } p \text { ). }
\end{aligned}
$$

It is possible to prove that if $\varphi \in C^{k}$, then $W^{s}(p)$ and $W^{u}(p)$ are $C^{k}$ manifolds.

We say that $x$ is a homoclinic point associated to $p$ if $x \in W^{s}(p) \cap W^{u}(p)$, and that $\varphi$ exibits a homoclinic tangency at $x$ associated to $p$ if $W^{s}(p)$ is tangent to $W^{u}(p)$ at $x$.

Let $\Lambda \subset M$. We say that $\Lambda$ is an hyperbolic set for $\varphi: M \rightarrow M$ if $\varphi(\Lambda)=\Lambda$ and there is a decomposition $T_{\Lambda} M=E^{s} \oplus E^{u}$ such that $\left.D \varphi\right|_{E^{s}}$ is uniformly contractive and $\left.D \varphi\right|_{E^{u}}$ is uniformly expansive. Given $x \in \Lambda$, we define:

$$
\begin{aligned}
& W^{s}(x)=\left\{y \in M \mid \lim _{n \rightarrow \infty} d\left(\varphi^{n}(y), \varphi^{n}(x)\right)=0\right\} \\
& W^{u}(x)=\left\{y \in M \mid \lim _{n \rightarrow-\infty} d\left(\varphi^{n}(y), \varphi^{n}(x)\right)=0\right\},
\end{aligned}
$$

which are $C^{k}$ manifolds. The union of these manifolds forms foliations $\mathcal{F}^{s}$ and $\mathcal{F}^{u}$ which can be extended to a neighbourhood of $\Lambda$ and which are leaf-wise $\varphi$-invariants.

The non-wandering set of $\varphi, \Omega(\varphi)$, is defined by:

$$
\begin{aligned}
\Omega(\varphi):= & \left\{x \in M \mid \forall \text { neighbourhood } U(x), \exists n \in \mathbf{Z}_{+}\right. \\
& \left.\operatorname{tq} \varphi^{n}(U(x)) \cap U(x) \neq \emptyset\right\} .
\end{aligned}
$$

Vol. 13, $n^{\circ}$ 6-1996. 
We say that $\varphi$ is hyperbolic if $\Omega(\varphi)$ is hyperbolic. A 1-parameter family $\left\{\varphi_{\mu}\right\}$ of diffeomorphisms of $M^{2}$ has an homoclinic $\Omega$ - explosion at $\mu=0$ if

i) for $\mu<0, \varphi_{\mu}$ is persistently hyperbolic;

ii) $\Omega\left(\varphi_{0}\right)=\Lambda \cup \mathcal{O}$, where $\Lambda$ is hyperbolic and $\mathcal{O}$ is an homoclinic tangency orbit associated to a fixed saddle point $p$, such that $W^{s}(p)$ and $W^{u}(p)$ have quadratic tangencies at the points of $\mathcal{O}$.

A Cantor set of the real line is a compact, with empty interior and whithout isolated points subset of $\mathbf{R}$.

Given $K \subset \mathbf{R}$ a Cantor set and $\mathcal{U}$ a finite covering of $K$ by open intervals $\mathcal{U}=\left(U_{i}\right)_{i \in I}$ we define $\operatorname{diam}(\mathcal{U})=\max \left\{\left|U_{i}\right|, \quad i \in I\right\}$, where $\left|U_{i}\right|$ is the lenght of $U_{i}$. Given $\alpha \in \mathbf{R}_{+}$, we define $H_{\alpha}(\mathcal{U})=\sum_{i \in I}\left|U_{i}\right|^{\alpha}$.

The Hausdorff $\alpha$-measure of $K$ is $m_{\alpha}(K)=\lim _{\varepsilon \rightarrow 0}\left(\underset{\substack{\inf \\ \operatorname{diam}(\mathcal{U})<\varepsilon}}{\operatorname{covers} K} H_{\alpha}(\mathcal{U})\right)$.

It is easy to see that there is an unique real number, the Hausdorff dimension of $K$, which we name $H D(K)$ such that, $\alpha<H D(K) \Rightarrow$ $m_{\alpha}(K)=+\infty$, and $\alpha>H D(K) \Rightarrow m_{\alpha}(K)=0$.

\section{I.1. Dynamically defined Cantor sets}

Let us consider a homoclinic tangency associated to a saddle point $p$ of a diffeomorphism $\varphi$ of a surface. Suppose that $p$ belongs to a basic set $\Lambda$ of saddle-type (a horseshoe). The intersection of the local stable manifold of $p$ and $\Lambda$ is a Cantor set which we will name $K^{s}$, and the intersection of the local unstable manifold of $p$ and $\Lambda$ is another Cantor set which we will name $K^{u}$.

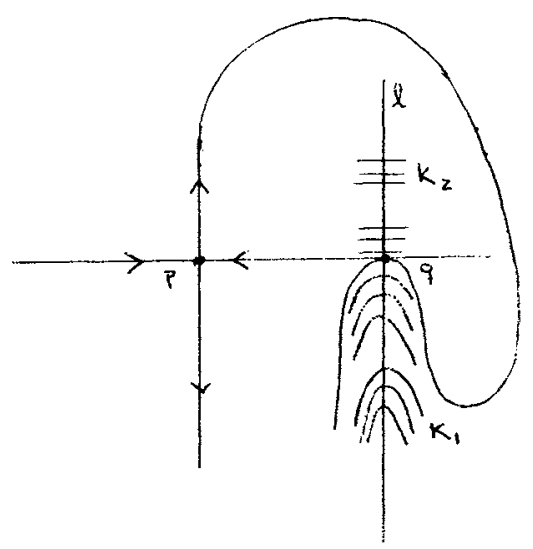


If $q$ is a point of tangency, unfolding the tangency by a generic family $\varphi_{\mu}\left(\varphi_{0}=\varphi\right)$ of diffeomorphisms, we will have a "line of tangencies" $\ell_{\mu}$ where the leaves of the foliations $\mathcal{F}_{\mu}^{s}$ and $\mathcal{F}_{\mu}^{u}$ are tangent. The projections of $K_{\mu}^{s}$ and $K_{\mu}^{u}$ (the continuations of $K^{s}$ and $K^{u}$ ) along leaves of the foliations over $\ell_{\mu}$ give Cantor sets diffeomorphic to $K_{\mu}^{s}$ and $K_{\mu}^{u}$ which we call $\left(K_{1}\right)_{\mu}$ and $\left(K_{2}\right)_{\mu}$, respectively. If $\left(K_{1}\right)_{\mu} \cap\left(K_{2}\right)_{\mu} \neq \emptyset$, there will be a new tangency between $W^{s}\left(\Lambda_{\mu}\right)$ and $W^{u}\left(\Lambda_{\mu}\right)$, and $\varphi_{\mu}$ will not be persistently hyperbolic.

With some generic hypothesis we can conclude (perhaps reparametrizing the family $\left.\left(\varphi_{\mu}\right)\right)$ that the pair $\left(\left(K_{1}\right)_{\mu},\left(K_{2}\right)_{\mu}\right)$ is close to the pair $\left(K_{1}, K_{2}+\mu\right)$, and then is natural to ask about the size of the set $\left\{\mu \mid K_{1} \cap\left(K_{2}+\mu\right) \neq \emptyset\right\}=: K_{1}-K_{2}=\left\{x-y \mid x \in K_{1}, y \in K_{2}\right\}$. We say that $K_{1}-K_{2}$ is the arithmetic difference between $K_{1}$ and $K_{2}$.

The Cantor sets that we will consider (and in particular the ones above) are of a special kind: the dynamically defined Cantor sets. We say that a Cantor set $K$ is dynamically defined if:

i) there are disjoint compact intervals $K_{1}, K_{2}, \ldots K_{r}$ such that $K \subset$ $K_{1} \cup \ldots \cup K_{r}$ and the boundary of each $K_{i}$ is contained in $K$;

ii) there is a $C^{1+\varepsilon}$ expanding map $\psi$ defined in a neighbourhood of $K_{1} \cup K_{2} \cup \ldots \cup K_{r}$ such that $\psi\left(K_{i}\right)$ is the convex hull of a finite union of some intervals $K_{j}$ satisfying:

ii.1 For each $i, 1 \leq i \leq r$ and $n$ sufficiently big, $\psi^{n}\left(K \cap K_{i}\right)=K$;

ii.2 $K=\cap_{n=0}^{\infty} \psi^{-n}\left(K_{1} \cup K_{2} \cup \ldots \cup K_{r}\right)$.

We say that $\left\{K_{1}, K_{2}, \ldots K_{\tau}\right\}$ is a Markov partition for $K$, and that $D=\cup_{i=1}^{r} K_{i}$ is the Markov domain of $K$.

If $K_{1}=[a, b]$ is the left interval of has of the Markov partition it is usual to suppose that $\left.\psi\right|_{K_{1}}: K_{1} \rightarrow K_{0}$ is surjective, where $K_{0}$ is the convex hull of $K$, and increasing. In this case, there is a diffeomorphism $\alpha: K_{0} \rightarrow \tilde{K}_{0}$ (where $\tilde{K}_{0}$ is an interval) with $\alpha^{\prime}(0)=1$ such that $\alpha \circ \psi_{1} \circ \alpha^{-1}$ is an affine map, because $\left|\psi_{1}^{\prime}(a)\right|>1$. In this case the set $\tilde{K}=\alpha(K)$ defined by the functions $\tilde{\psi}_{i}=\alpha \circ \psi_{i} \circ \alpha^{-1}$ is auto-similar (has $\tilde{\psi}_{1}$ affine), and is called the linearized of $K$.

For a more careful discussion of the relationship between dynamically defined Cantor sets, see [PT2].

The proof of the fact that if $H D(K)+H D\left(K_{2}\right)<1$ then $K_{1}-K_{2}$ has measure zero, and the study of dynamical consequences of this result (prevalence of hyperbolicity near $\mu=0$ ) may be found in [PT1] and in [PT2]. Let we sketch the proof: 
Consider the map $f: \mathbf{R}^{2} \rightarrow \mathbf{R}, f(x, y)=x-y$. Then $K_{1}-K_{2}=$ $f\left(K_{1} \times K_{2}\right)$, and it is possible to prove (see [PT2]) that if $K_{1}$ and $K_{2}$ are dynamically defined Cantor sets, then $H D\left(K_{1} \times K_{2}\right)=$ $H D\left(K_{1}\right)+H D\left(K_{2}\right)$, which we suppose to be less than 1 . Therefore, $K_{1}-K_{2}=f\left(K_{1} \times K_{2}\right)$ is a Lipschitz image of a set with Hausdorff dimension less than one, and so has Hausdorff dimension less than one, and therefore Lebesgue measure zero.

\section{I.2. Intersections of Cantor sets and arithmetic differences}

As we have seen in I.1, the study of homoclinic bifurcations leads naturally to the study of intersections of Cantor sets, and to the study of arithmetic differences of Cantor sets. About this, there is Palis' conjecture mentioned before, according to which the arithmetic difference of two dynamically defined Cantor sets generically either have measure zero, or Contains an interval. There is an example by A. Sannami [S] of a dynamically defined Cantor set $K$ such that $K-K$ is a Cantor set with positive measure. Bamón, Plaza and Vera also discuss this kinds of examples in [BPV]. These examples are very rigid, and the Palis' conjecture remains. For affine Cantor sets (which we will define later) there is no counter example.

In this work we shall study a concept as closely related to homoclinic bifurcations as that of arithmetic difference: the concept of stable intersections. We say that two dynamically defined Cantor sets $K_{1}$ and $K_{2}$ have stable intersection if there is a neighbourhood of the pair $\left(K_{1}, K_{2}\right)$ (in some apropriate topology; here we shall generally work with the $C^{1+\varepsilon}$ topology, that we shall define later) such that for every pair $\left(\tilde{K}_{1}, \tilde{K}_{2}\right)$ in this neighbourhood we have $\tilde{K}_{1} \cap \tilde{K}_{2} \neq \emptyset$.

Here we will generally work with the topology $C^{1+\varepsilon}$ in which a Cantor set $K$ defined by an expansive function $\psi$ with Markov partition $\left\{K_{1}, K_{2}, \ldots K_{r}\right\}$ is close to a Cantor set $\tilde{K}$ with Markov partition $\left.\tilde{K}_{1}, \tilde{K}_{2}, \ldots \tilde{K}_{s}\right\}$ if and only if $r=s$, the extremes of $K_{i}$ are near the corresponding extremes of $\tilde{K}_{i}, i=1,2, \ldots, r$ and, supposing $\psi \in C^{1+\varepsilon}$ with Hölder constant $C$, we must have $\tilde{\psi} \in C^{1+\tilde{\varepsilon}}$ with Hölder constant $\tilde{C}$ such that $(\tilde{C}, \tilde{\varepsilon})$ is near $(C, \varepsilon)$ and $\tilde{\psi}$ is close to $\psi$ in the $C^{1}$ topology.

This is a natural topology because, on one hand it assures the control of the distortion (see [PT2]). In particular, if $K$ have small distortion, then so does $\tilde{K}$ (for instance, this holds when $K$ is affine). On the other hand, the sets $K^{s}$ and $K^{u}$ associated to a diffeomorphism $\varphi \in C^{2}$ depend continuously of $\varphi$ in the $C^{1+\varepsilon}$ topology. 
There are relations between the concepts of aritmetic difference and stable intersection. For instance, if $K_{1}$ intersects $K_{2}$ stably then $t \in \operatorname{int}\left(K_{1}-K_{2}\right)$. In the direction of the reciprocal we have the following result:

THEOREM I.1. - Suppose that there is an open set $U$ of pairs of Cantor sets such that if $\left(K_{1}, K_{2}\right) \in U$ and $t \in \mathbf{R}$ then $K_{1}$ does not have stable intersection with $\left(K_{2}+t\right)$. Then there is a residual set $R \subset U$ such that $\left(K_{1}, K_{2}\right) \in R \Rightarrow \operatorname{int}\left(K_{1}-K_{2}\right)=\emptyset$; that is the interior of $K_{1}-K_{2}$ is empty.

Proof. - Let $\left\{r_{1}, r_{2}, \ldots, r_{n}, \ldots\right\}$ be an enumeration of the rational numbers, and let $U_{n}=\left\{\left(K_{1}, K_{2}\right) \in U \mid K_{1} \cap\left(K_{2}+r_{n}\right)=\emptyset\right\}$. By this hypothesis, $U_{n}$ is dense, and clearly $U_{n}$ is open. Thus, $R:=\cap_{n=1}^{\infty} U_{n}$ is residual, and $\left(K_{1}, K_{2}\right) \in R \Rightarrow\left(K_{1}-K_{2}\right) \cap \mathbf{Q}=\emptyset \Rightarrow \operatorname{int}\left(K_{1}-K_{2}\right)=$ $\emptyset$.

Remark. - The results above are valid in any $C^{k}$ topology, $k \geq 1$.

We recall that a dynamically defined Cantor set is affine if the functions $\psi_{i}:=\left.\psi\right|_{K_{i}}$ that define $K$ are affine and surjective. This implies that $K$ is similar to $K \cap K_{i}, 1 \leq i \leq r$. If we do not suppose the functions $\psi_{i}$ to be surjective, we say that $K$ is a generalized affine Cantor set.

Let us fix a notation for a certain type of affine Cantor set that will be often used in examples.

Frequently, when we mention an affine Cantor set of the type

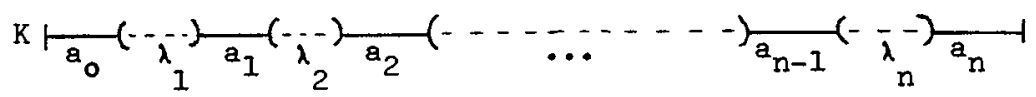

we mean the Cantor set with this Markov domain defined by increasing and surjective affine maps.

The study of stable intersections of Cantor sets has more immediate applications to dynamics than the study of arithmetic differences. As an example, there is a theorem by Marstrand that implies that if $H D\left(K_{1}\right)+H D\left(K_{2}\right)>1$, then for almost every $\lambda \in \mathbf{R}, K_{1}-\lambda K_{2}$ has positive Lebesgue measure (see [PT2]); and there is a theorem by Palis and Yoccoz, according to which if $H D\left(K^{s}\right)+H D\left(K^{u}\right)>1$, then generically $\mu=0$ is not a point of total density of hyperbolicity of $\left(\varphi_{\mu}\right)$ (see [PY]). This theorem is, however, very difficult, and by no means a trivial consequence of Marstrand's theorem.

We will now see an application of stable intersections to dynamics.

THEOREM I.2. - In the case of the homoclinic bifurcation, if there is $t \in \mathbf{R}$ such that $\left(\tilde{K}_{1}\right)_{0}$ intersects $\left(\left(\tilde{K}_{2}\right)_{0}+t\right)$ stably, where $\left(\tilde{K}_{1}\right)_{0}$ 
and $\left(\tilde{K}_{2}\right)_{0}$ are linearized of $\left(K_{1}\right)_{0}$ and $\left(K_{2}\right)_{0}$, respectively, then the set $\left\{\mu>0 \mid\left(K_{1}\right)_{\mu} \cap\left(K_{2}\right)_{\mu} \neq \emptyset\right\}$ contains an open set with positive density at $\mu=0$.

Proof. - Let $\left(\tilde{K}_{1}\right)_{\mu}$ and $\left(\tilde{K}_{2}\right)_{\mu}$ be the linearized of $\left(K_{1}\right)_{\mu}$ and $\left(K_{2}\right)_{\mu}$, respectively. Then $\left(\tilde{K}_{1}\right)_{\mu}$ and $\left(\tilde{K}_{2}\right)_{\mu}$ are diffeomorphic to $K_{\mu}^{s}$ and $K_{\mu}^{u}$, and we have $\left(\lambda_{1}\right)_{\mu}\left(\tilde{K}_{1}\right)_{\mu} \subset\left(\tilde{K}_{1}\right)_{\mu}$ and $\left(\lambda_{2}\right)_{\mu}^{-1}\left(\tilde{K}_{2}\right)_{\mu} \subset\left(\tilde{K}_{2}\right)_{\mu}$, where $\left(\lambda_{1}\right)_{\mu}$ and $\left(\lambda_{2}\right)_{\mu}$ are the eigenvalues of the diffeomorphism $\varphi_{\mu}$. By the differenciability of the family $\left(\varphi_{\mu}\right)$ relative to $\mu$ there is a constant $C>0$ such that $\left|\left(\lambda_{i}\right)_{\mu}-\lambda_{i}\right| \leq C|\mu|, 1=1,2$, for $|\mu|$ small. Besides, there are $C^{1+\varepsilon}$ diffeomorphisms $\left(h_{1}\right)_{\mu}$ and $\left(h_{2}\right)_{\mu}$ which depend continuously in the $C^{1+\varepsilon}$ topology on $\mu$ such that $\left(K_{1}\right)_{\mu}=\left(h_{1}\right)_{\mu}\left(\left(\tilde{K}_{1}\right)_{\mu}\right)$ and $\left(K_{2}\right)_{\mu}=\left(h_{2}\right)_{\mu}\left(\left(\tilde{K}_{2}\right)_{\mu}\right)$, with $\left(h_{1}\right)_{0}^{\prime}(\mu)=\left(h_{2}\right)_{0}^{\prime}=1$. We suppose, after reparametrization of $\ell_{\mu}$, that $\left(\tilde{K}_{1}\right)_{\mu} \subset(-\infty, 0],\left(\tilde{K}_{2}\right)_{\mu} \subset[0, \infty), 0 \in$ $\left(\tilde{K}_{1}\right)_{\mu} \cap\left(\tilde{K}_{2}\right)_{\mu}, h_{1}(0)=\mu$ and $h_{2}(0)=0$.

By the hypothesis, there is an $\varepsilon>0$ such that if $\left(K, K^{\prime}\right)$ is near $\left(\left(\tilde{K}_{1}\right)_{0},\left(\tilde{K}_{2}\right)_{0}+t\right)$, with $\left|t^{\prime}-t\right|<\varepsilon$ then $K$ intersects $K^{\prime}$ stably. Suppose $\left|\lambda_{1}\right|<1,\left|\lambda_{2}\right|>1$ and let $m, n \in \mathbf{N}$ be such that $\left|\lambda_{1}^{m} \lambda_{2}^{n}-1\right|$ is sufficiently small. Then, if $\mu \in\left(\lambda_{2}^{-n}(t-\varepsilon), \lambda_{2}^{-n}(t+\right.$ $\varepsilon)$ ), where $n$ is large enough, we will have $\left(K_{1}\right)_{\mu} \cap\left(K_{2}\right)_{\mu} \neq \emptyset$. $\left(\lambda_{1}\right)_{\mu}^{m}\left(\tilde{K}_{1}\right)_{\mu} \subseteq\left(\tilde{K}_{1}\right)_{\mu}$ and $\left(\lambda_{2}\right)_{\mu}^{-n}\left(\tilde{K}_{2}\right)_{\mu} \subseteq\left(\tilde{K}_{2}\right)_{\mu}$. It is enough to prove that $\left(h_{1}\right)_{\mu}\left(\left(\lambda_{1}\right)_{\mu}^{m}\left(\tilde{K}_{1}\right)_{\mu}\right) \cap\left(h_{2}\right)_{\mu}\left(\left(\lambda_{2}\right)_{\mu}^{-n}\left(\tilde{K}_{2}\right)_{\mu}\right) \neq \emptyset$, or, equivalently, that $\left.\left(\lambda_{1}\right)_{\mu}^{-m} \cdot\left(h_{1}\right)_{\mu}\left(\left(\lambda_{1}\right)_{\mu}^{m}\left(\tilde{K}_{1}\right)_{\mu}\right) \cap\left(\lambda_{1}\right)_{\mu}^{-m} \cdot\left(h_{2}\right)_{\mu}\left(\left(\lambda_{2}\right)\right) \mu^{-m}\left(\tilde{K}_{2}\right)_{\mu}\right) \neq \emptyset$, but this follows from the fact that the first set is close to $\left(\tilde{K}_{1}\right)_{0}+\lambda_{1}^{-m} \mu$ and the second is close to $\left(\tilde{K}_{2}\right)_{0}$, because $\left(h_{1}\right)_{\mu}(x) \sim x+\mu$ and $\left(h_{2}\right)_{\mu}(x) \sim x$ for $x$ near 0 , and also $\lambda_{1}^{m} /\left(\lambda_{1}\right)_{\mu}^{m}$ and $\lambda_{\lambda}^{n} /\left(\lambda_{2}\right)_{\mu}^{n}$ are close to 1 . This last affirmation follows from $\left|\left(\lambda_{i}\right)_{\mu}-\lambda_{i}\right| \leq K|\mu| \leq K^{\prime \prime} \lambda_{2}^{-n} \leq K^{\prime \prime} \lambda_{1}^{n}$, where $K, K^{\prime}$ and $K^{\prime \prime}$ are constants. Then, for instance, $\left(\left(\lambda_{1}\right)_{\mu} / \lambda_{1}\right)^{m} \leq\left(1+K^{\prime \prime} \lambda^{m-1}\right)^{m} \sim 1$, because $m \cdot \lambda_{1}^{m-1} \rightarrow 0$ when $m \rightarrow \infty$.

To conclude the proof it is enough to show that:

(*) there is $N \in \mathbf{N}$ such that for each $K \in \mathbf{N}$ there is $m \in \mathbf{N}$ with $K \leq m<K+N$ and $n \in \mathbf{N}$ with $\left|\lambda_{1}^{m} \lambda_{2}^{n}-1\right|$ suficiently small.

And (*) is a consequence of:

(**) there are $N^{\prime}, N^{\prime \prime} \in \mathbf{N}$ such that $\lambda_{1}^{N^{\prime}} \lambda_{2}^{N^{\prime \prime}}$ is very close to 1 .

Note that $\left(^{* *}\right)$ is equivalent to obtaining $N^{\prime}, N^{\prime \prime} \in \mathbf{N}$ such that $N^{\prime} \log \lambda_{1}+N^{\prime \prime} \log \lambda_{2}$ is close to 0 , which it is possible if $\log \lambda_{1} / \log \lambda_{2}$ is irrational by Dirichlet's theorem and if $\log \lambda_{1} / \log \lambda_{2}=-p / q, p, q \in \mathbf{N}$ we can take $N^{\prime}=q, N^{\prime \prime}=p$. To deduce $(*)$ from $(* *)$ observe that $\lambda_{1}^{N^{\prime}} \lambda_{2}^{N^{\prime \prime}}=\lambda_{1}^{\tau}$ with $|\tau|$ very small. We have 2 cases:

- $\tau=0$ : Take $N=N^{\prime}, m=K N^{\prime}, n=K N^{\prime \prime}(K \in \mathbf{N})$ 
- $\tau \neq 0$ : take $\tilde{N}=\left[\frac{1}{|\tau|}\right]+1, \quad N=N^{\prime} \tilde{N}, \quad m=N^{\prime}\left|\left[\frac{K}{\tau}\right]\right|-K \frac{\tau}{|\tau|}$ and $n=N^{\prime \prime}\left|\left[\frac{K}{\tau}\right]\right|(K \in \mathbf{N})$.

Remark 1. - If $\frac{\log \lambda_{1}}{\log \lambda_{2}} \notin \mathbf{Q}$, it is enough to suppose there exists $\lambda>0$ and $t \in \mathbf{R}$ such that $\left(\tilde{K}_{1}\right)_{0}$ intersects $\left(\lambda\left(\tilde{K}_{2}\right)_{0}+t\right)$ stably.

Remark 2. - The hypothesis of the Theorem 1.2 are open, and as a consequence of the demonstration we can obtain an uniform positive estimate in a neighbourhood of the family $\left(\varphi_{\mu}\right)$ to $\liminf _{\delta \rightarrow 0}$ $\frac{m\left(\left\{\mu \in[0, \delta] \mid\left(K_{1}\right)_{\mu} \cap\left(K_{2}\right)_{\mu} \neq \emptyset\right\}\right)}{\delta}$.

We say that the pair of Cantor sets $\left(K_{1}, K_{2}\right)$ have extremal stable intersection if the right extreme of $K_{1}$ coincides with the left one of $K_{2}$, and if $\left(\tilde{K}_{1}, \tilde{K}_{2}\right)$ is close to $\left(K_{1}, K_{2}\right)$, and the support intervals of $\tilde{K}_{1}$ and $\tilde{K}_{2}$ intersects their $\tilde{K}_{1} \cap \tilde{K}_{2} \neq \emptyset$. This implies in particular that $\left(K_{1}+\varepsilon, K_{2}\right)$ has stable intersection, for any $\varepsilon>0$ small.

The importance of this concept to the study of homoclinic bifurcations is that if the pair $\left(\left(K_{1}\right)_{0},\left(K_{2}\right)_{0}\right)$ have extremal stable intersection then $\left\{\mu>0 \mid\left(K_{1}\right)_{\mu} \cap\left(K_{2}\right)_{\mu} \neq \emptyset\right\}$ contains an interval $[0, \delta]$ for some $\delta>0$.

We shall study in this work conditions that imply stable intersections and extremal stable intersections of dynamically defined Cantor sets.

We end this section with a problem that we consider to be very important in our context: are the families $\left\{\varphi_{\mu}\right\}$ satisfying the condition in Theorem I.2 dense in the region $\left\{\left(\varphi_{\mu}\right) \mid H D\left(K^{s}\right)+H D\left(K^{u}\right)>1\right\}$ ? This is, in a certain sense, a stronger version of Palis' conjecture, and, if it is true, we will have a stronger version of the results of Palis-Yoccoz paper [PY].

\section{THICKNESS AND LATERAL THICKNESS}

\section{II.1. Definitions, examples and consequences of stable intersections}

Definition. - A gap of a Cantor set is a connected component of its complement.

Given an $U$ gap of a Cantor set $K$, we associate to it the intervals $L_{U}$ and $R_{U}$, which are the intervals to its left and its right that separate it from the closest larger gaps.

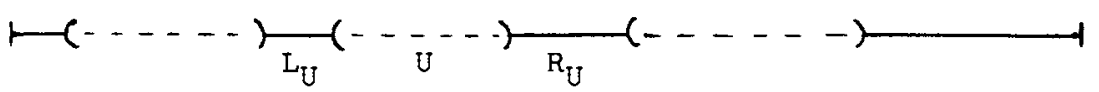


We define $\tau_{R}(U)=\frac{\left|R_{U}\right|}{|U|}, \tau_{L}(U)=\frac{\left|L_{U}\right|}{|U|}$, $\tau_{R}(K)=\inf \left\{\tau_{R}(U) \mid U\right.$ bounded gap of $\left.K\right\}$, the right thickness of $K$; $\tau_{L}(K)=\inf \left\{\tau_{L}(U) \mid U\right.$ bounded gap of $\left.K\right\}$, the left thickness of $K$, and $\tau(K)=\min \left\{\tau_{R}(K), \tau_{L}(K)\right\}$, the thickness of $K$.

Remark. - Given a Cantor set $K$, a presentation of $K$ is an enumeration $\left\{U_{1}, U_{2}, \cdots\right\}$ of its limited gaps. We can define intervals $L_{U}$ and $R_{U}$ as being the intervals between $U$ and the nearest gaps of indexes smaller than $U$. In an case we are using the presentation by order of the gaps'size, which maximizes the thickness $\tau(K)$. See [PT2].

The importance of the thicknesses derives from in the following result, which is an adaptation of the "gap lemma" ([PT2]).

PROPOSITION II.1. - Given $K_{1}$ Cantor sets, if $\tau_{R}\left(K_{1}\right) \cdot \tau_{L}\left(K_{2}\right)>1$ and $\tau_{L}\left(K_{1}\right) \cdot \tau_{R}\left(K_{2}\right)>1$, then either $K_{1}$ is contained in a gap of $K_{2}$ or $K_{2}$ is contained in a gap of $K_{1}$ or $K_{1} \cap K_{2} \neq \emptyset$.

Remark. - This holds in particular if $\tau\left(K_{1}\right) \cdot \tau\left(K_{2}\right)>1$, which is the hypothesis of the known gap lemma.

Proof. - Consider the case in which none of the $K_{i}$ is contained in a gap of the other. There is, then, a pair of linked gaps, such as in the figures below:

a)

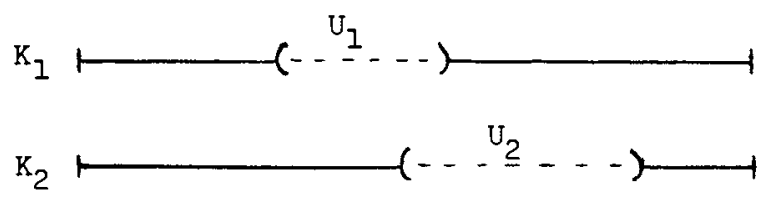

Since $\tau_{R}\left(U_{1}\right) \tau_{L}\left(U_{2}\right)>1$, we have $\left|R_{U_{1}}\right|>\left|U_{2}\right|$ or $\left|L_{U_{2}}\right|>\left|U_{1}\right| \Rightarrow$ a new pair of gaps exists like in smaller than the pair $\left(U_{1}, U_{2}\right)$

b)

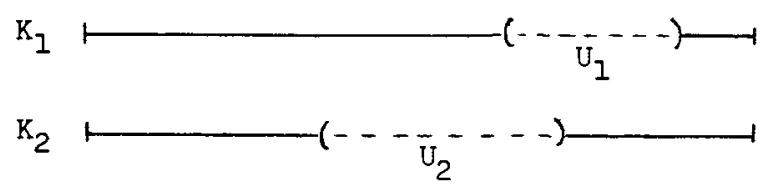

As $\tau_{L}\left(U_{1}\right) \tau_{R}\left(U_{2}\right)>1$, we have $\left|L_{U_{1}}\right|>\left|U_{2}\right|$ or $\left|R_{U_{2}}\right|>\left|U_{1}\right| \Rightarrow$ a new pair of gaps exists like in a) smaller than the pair $\left(U_{1}, U_{2}\right)$.

In any case, we obtain a smaller pair of gaps, for instance in the sense that the sum of the lenghts of its gaps decrease. Using repeatedely the same 
argument we obtain a seguence of pairs of gaps converging to a point that necessarily belongs to $K_{1} \cap K_{2}$.

We can define, for $p \in K, \tau_{\text {loc }}(K, p)=\limsup _{\varepsilon \rightarrow 0} \tau(\overline{K \cap(p-\varepsilon, p+\varepsilon)})$ and similar $\left(\tau_{R}\right)_{\text {loc }}(K, p)$ and $\left(\tau_{L}\right)_{\text {loc }}(K, p)$. For dynamically defined Cantor sets, $\tau_{\text {loc }}(K, p)$ does not depend on $p$ (see [PT2]), and the same proof shows that $\left(\tau_{R}\right)_{\text {loc }}(K, p)$ and $\left(\tau_{L}\right)_{\text {loc }}(K, p)$ do not depend on $p$ either. Let us therefore, call them $\tau_{\text {loc }}(K),\left(\tau_{R}\right)_{\text {loc }}(K)$ and $\left(\tau_{L}\right)_{\text {loc }}(K)$.

Proposition II-1. - implies that if $\left(\tau_{R}\right)\left(K_{1}\right) \cdot\left(\tau_{L}\right)\left(K_{2}\right) \geq 1$ and $\left(\tau_{L}\right)\left(K_{1}\right) \cdot\left(\tau_{R}\right)\left(K_{2}\right) \geq 1$ then $K_{1}-K_{2}$ contains an interval.

As a result, if $\left(\tau_{R}\right)_{\mathrm{loc}}\left(K_{1}\right) \cdot\left(\tau_{L}\right)_{\mathrm{loc}}\left(K_{2}\right)>1$ and $\left(\tau_{L}\right)_{\mathrm{loc}}\left(K_{1}\right)$. $\left(\tau_{R}\right)_{\text {loc }}\left(K_{2}\right)>1$, then $K_{1}-K_{2}$ contains an interval.

For this last result we need strict inequalities. Indeed, in Sannami's example of a dynamically defined Cantor set $K$ with $\lambda(K-K)>0$ and $\operatorname{int}(K-K)=\emptyset$ have $\tau_{\text {loc }}(K)=1$. (See example III.3).

Also as a result of Proposition II-1, if $\tau_{R}\left(K_{1}\right) \cdot \tau_{L}\left(K_{2}\right)>1$ and $\tau_{L}\left(K_{1}\right) \cdot \tau_{R}\left(K_{2}\right)>1$, and if $\tau_{R}$ and $\tau_{L}$ are continuous in $K_{1}$ and $K_{2}$, then, for every $t \in \mathbf{R}$ such that $K_{1}$ and $K_{2}+t$ are linked, $K_{1}$ intersects $K_{2}+t$ stably.

Thickness $\tau(K)$ is continuous in the $C^{1+\varepsilon}$ topology (see [PT2]). The same thing does not occur with lateral thicknesses: as be seen in the following example.

Example II.1.1. - In the set

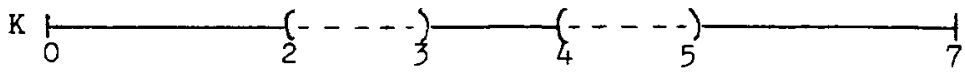

the lateral thicknesses are not continuous.

In fact we have, next to $K$, sets
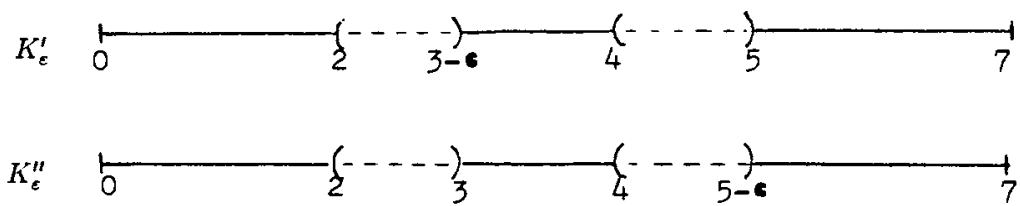

$K_{\varepsilon}^{\prime} K_{\varepsilon}^{\prime \prime}$ for which $\tau_{R}\left(K_{\varepsilon}^{\prime}\right)=\frac{1+\varepsilon}{1-\varepsilon}(\rightarrow 1$ when $\varepsilon \rightarrow 0)$ and $\tau_{L}\left(K_{\varepsilon}^{\prime}\right)=$ $\frac{1}{1-\varepsilon}(\rightarrow 2$ when $\varepsilon \rightarrow 0), \tau_{R}\left(K_{\varepsilon}^{\prime}\right)=\frac{2+\varepsilon}{1-\varepsilon}(\rightarrow 2$ when $\varepsilon \rightarrow 0)$ and 
$\tau_{L}\left(K_{\varepsilon}^{\prime}\right)=\frac{1}{1-\varepsilon}(\rightarrow 1$ when $\varepsilon \rightarrow 0)$, which shows that $\tau_{R}$ and $\tau_{L}$ are discontinuous in $K$.

We shall prove in II.2 that there is an open and dense set $U$ in the $C^{1+\varepsilon}$ topology where the lateral thicknesses are continuous. We shall also prove that the lateral thicknesses are continuous in affine Cantor sets of the type

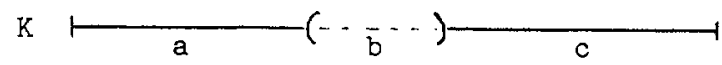

for which the right thickness $\tau_{R}(K)=\frac{c}{b}$ and the left $\tau_{L}(K)=\frac{a}{b}$.

Therefore, if $\frac{a_{1} c}{b_{1} b}>1$ e $\frac{c_{1} a}{b_{1} b}>1$, the sets
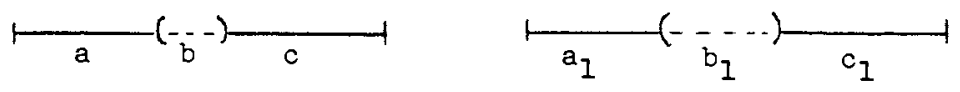

have stable intersection whenever they are linked, and its arithmetic difference will contain an interval. This phenomenon being stable among dynamically defined Cantor sets.

There is an inequality whose proof can be found in [PT2] which states that $H D(K) \geq \log 2 / \log \left(2+\frac{1}{\tau(K)}\right)$, which implies that if $\tau(K) \geq 1$, then $H D(K) \geq \log 3 / \log 2>0.6$. Therefore, if $K_{1}$ and $K_{2}$ have Hausdorff dimension 0.6 each, the product of their thicknesses is smaller than 1 , and consequently the test of thickness cannot guarantee the existence of stable intersection between $K_{1}$ and $K_{2}$.

However, the notion of lateral thickness makes it possible to prove the next result.

THEOREM II-1.2. - Given $h_{1}$, and $h_{2}$ in the interval $(0,1)$ with $h_{1}+h_{2}>1$, there are affine Cantor sets $K_{1}$ and $K_{2}$ with Hausdorff dimensions $h_{1}$ and $h_{2}$, respectively, that intersect stably. Besides we can obtain that $\left(K_{1}, K_{2}\right)$ and $\left(K_{2}, K_{1}+1\right)$ have extremal stable intersection.

Proof. - We shall look for examples of the type

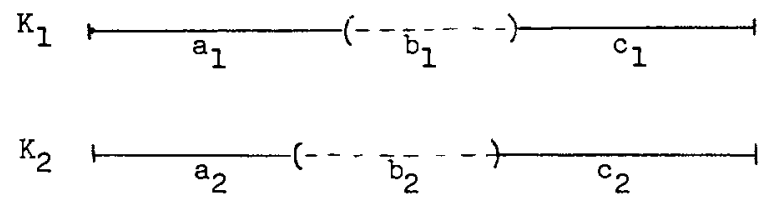

In a set

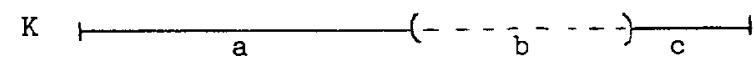


with a $a+b+c=1$ we have $H D(K)=\lambda$ where $\lambda$ is the only real number such that $a^{\lambda}+c^{\lambda}=1$ (see [PT2]), $\tau_{R}(K)=c / b$ and $\tau_{L}(K)=a / b$.

Hence, if we have $\frac{a_{1} c_{2}}{b_{1} b_{2}}>1$ and $\frac{a_{2} c_{1}}{b_{1} b_{2}}>1,\left(K_{1}, K_{2}\right)$ will have stable intersection, provided they are linked. Besides, when in the position where the extremes touch, $\left(K_{1}, K_{2}\right)$ well have extremal stable intersection.

We shall try to obtain examples like the one above, with $a_{1}+b_{1}+c_{1}=$ $a_{2}+b_{2}+c_{2}=1$ and $a_{1} c_{2}=a_{2} c_{1}$ (that is, $\frac{c_{1}}{a_{1}}=\frac{c_{2}}{a_{2}}=: r$ ), so that the conditions $\frac{a_{1} c_{2}}{b_{1} b_{2}}>1$ and $\frac{a_{2} c_{1}}{b_{1} b_{2}}>1$ are equivalent. Given $h_{1}$ and $h_{2}$ in the interval $(0,1)$ with $h_{1}+h_{2}>1$, we shall obtain these examples with $H D\left(K_{1}\right)=h_{1}$ and $H D\left(K_{2}\right)=h_{2}$, that is, $a_{1}^{h_{1}}+c_{1}^{h_{1}}=1$ and $a_{2}^{h_{2}}+c_{2}^{h_{2}}=1$.

For a Cantor set

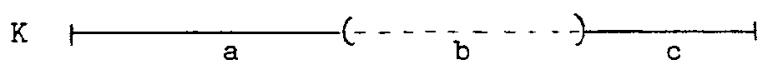

with $c=r a, a+b+c=1$ and $H D(K)=h$ we must have $a^{h}+(r a)^{h}=1 \Rightarrow a=\frac{1}{\left(1+r^{h}\right)^{1 / h}}, c=\frac{r}{\left(1+r^{h}\right)^{1 / h}}$ and $b=\frac{\left(1+r^{h}\right)^{1 / h}-1-r}{\left(1+r^{h}\right)^{1 / h}}$. In our case, we want $a_{1} c_{2}>b_{1} b_{2}\left(\Leftrightarrow a_{2} c_{1}>b_{1} b_{2}\right)$, and thus our problem is to find

$r>0$ satisfying

$$
\begin{aligned}
& \frac{1}{\left(1+r^{h_{1}}\right)^{1 / h_{1}}} \cdot \frac{r}{\left(1+r^{h_{2}}\right)^{1 / h_{2}}} \\
& \quad>\frac{\left(\left(1+r^{h_{1}}\right)^{1 / h_{1}}-1-r\right)}{\left(1+r^{h_{1}}\right)^{1 / h_{1}}} \cdot \frac{\left(\left(1+r^{h_{2}}\right)^{1 / h_{2}}-1-r\right)}{\left(1+r^{h_{2}}\right)^{1 / h_{2}}} \Leftrightarrow
\end{aligned}
$$

$$
r>\left(\left(1+r^{h_{1}}\right)^{1 / h_{1}}-1-r\right)\left(\left(1+r^{h_{2}}\right)^{1 / h_{2}}-1-r\right) .
$$

We have

$$
\begin{aligned}
& \lim _{r \rightarrow 0_{+}} \frac{\left(\left(1+r^{h}\right)^{1 / h}-1-r\right)}{r^{h}}=\frac{1}{h}, \\
& \lim _{r \rightarrow 0_{+}} \frac{\left(\left(1+r^{h_{1}}\right)^{1 / h_{1}}-1-r\right)\left(\left(1+r^{h_{2}}\right)^{1 / h_{2}}-1-r\right)}{r} \\
&=\lim _{r \rightarrow 0_{+}} \frac{\left(\left(1+r^{h_{1}}\right)^{1 / h_{1}}-1-r\right)}{r^{h_{1}}} \\
& \quad \cdot \lim _{r \rightarrow 0_{+}} \frac{\left.\left(1+r^{h_{2}}\right)^{1 / h_{2}}-1-r\right)}{r^{h_{2}}} \cdot \lim _{r \rightarrow 0_{+}} \frac{r^{h_{1}+h_{2}}}{r}=\frac{1}{h_{1}} \cdot \frac{1}{h_{2}} \cdot 0=0
\end{aligned}
$$

Vol. $13, n^{\circ} 6-1996$. 
since $h_{1}+h_{2}>1$, and thus, if $r$ is sufficiently small, the right side of $(*)$ is less than the left one. Thus, for every $r$ sufficiently small, we obtain examples as desired

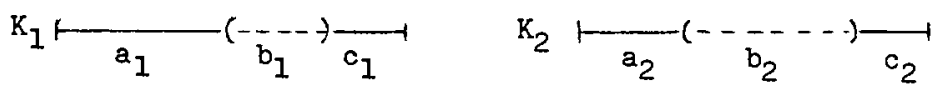

$$
\begin{aligned}
& a_{i}=\frac{1}{\left(1+r^{h_{i}}\right)^{1 / h_{i}}}, \quad c_{i}=\frac{r}{\left(1+r^{h_{i}}\right)^{1 / h_{i}}}, \quad b_{i}=1-a_{i}-c_{i} \text {, for } i=1,2
\end{aligned}
$$

Remark. - In the example IV.6 we will see another proof of this theorem.

\section{II.2. Continuity of the lateral thicknesses}

We saw in the example 1 of this section that the lateral thicknesses are not always continuous. However, there is an open and dense subset, in the $C^{1+\varepsilon}$ topology of dynamically defined Cantor sets whose elements have these thicknesses varying continuously. To see this we shall prove that there is an open and dense subset of dynamically defined Cantor sets $U$ in this topology such that if $K \in U$ then there exists $\delta>0$ such that if $U_{1} \neq U_{2}$ are gaps of $K$ and if there is no gaps $V$ between $U_{1}$ and $U_{2}$ with $|V|>2 \max \left\{\left|U_{1}\right|,\left|U_{2}\right|\right\}$ then:

$$
1+\delta<\left|U_{2}\right| /\left|U_{1}\right| \quad \text { or } \quad\left|U_{2}\right| /\left|U_{1}\right|<1-\delta
$$

Such sets are points of continuity of the lateral thickness. In fact, in this case, given a gap $U$ of $K$ and a gap $\tilde{U}$ corresponding to $U$ in a $\tilde{K}$ close to $K$, the gaps near $U$ large than $U$ which appear in the definition of thickness correspond dynamically to the gaps near $\tilde{U}$ larger than $\tilde{U}$. This, together with the arguments of distortion of used in the proof of the continuity of thickness in [PT2], implies that the lateral thicknesses are continuous in a dense open set.

To prove the density of condition $(*)$, we shall use the

LEMMA II.2.1. - Generalized affine Cantor sets are "dense" in the topology $C^{1+\varepsilon}$ in the sense that any $K$ can be approximated by a $\tilde{K}$ that coincides as a subset of the straight line with a generalized affine Cantor set (but it does not have to be defined by the same functions). 
Proof. - Consider an advanced step in the construction of $K$ where $K$ is a given Cantor set. The functions that define $K$ will be replaced by functions that coincide with the preceding in the extremities of the bridges of this stage, being affine in these bridges, remaining in class $C^{1}$.
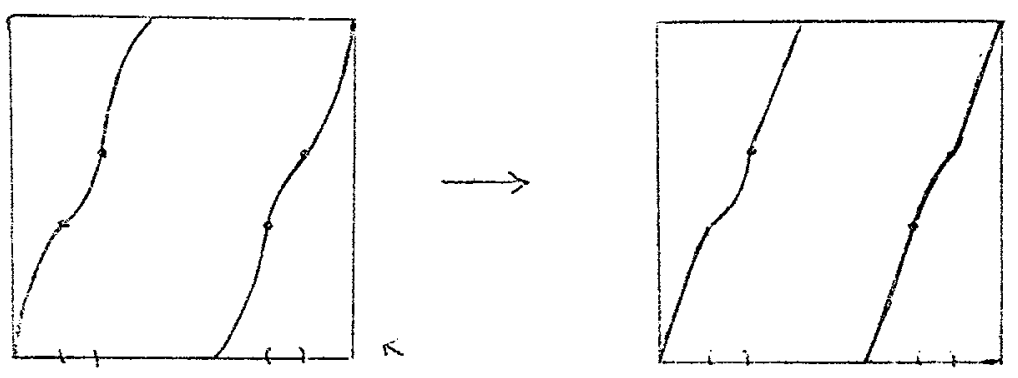

We claim that, if we do this in an advanced stage of the construction of $K$ we can obtain a $\tilde{K}$ set close to $K$ in the topology $C^{1+\varepsilon}$, and which coincides with an generalized affine set. For that purpose observe that if $I_{1}$ and $I_{2}$ are consecutive bridges and $\psi \in C^{1+\varepsilon},\left|\psi^{\prime}(x)-\psi^{\prime}(y)\right|<C|x-y|^{\varepsilon} \forall x, y$, and if $U$ is the gap between $I_{1}$ and $I_{2}$ then there is a constant $A$ with $|U| \geq A\left|I_{j}\right| j=1,2$, by limited distortion. By the mean value theorem, when we linearize (render affine) the function $\psi$ in $I_{1}$ and $I_{2}$ we shall obtain segments of inclination $\psi^{\prime}\left(c_{j}\right), c_{j} \in I_{j}, j=1,2$. We want to extend the function $\tilde{\psi}$ thus obtained to $U$. We shall do this by extending the derivative of $\tilde{\psi}$. Let $U=(a, b), c=\frac{a+b}{2}$. Suppose $\tilde{\psi} \equiv \lambda_{j}:=\psi^{\prime}\left(c_{j}\right)$ in $I_{j}$. We make $\tilde{\psi}^{\prime}$ affine in $(a, c)$ and $(c, b)$, assuming the value $\lambda_{3}$ in $c$.

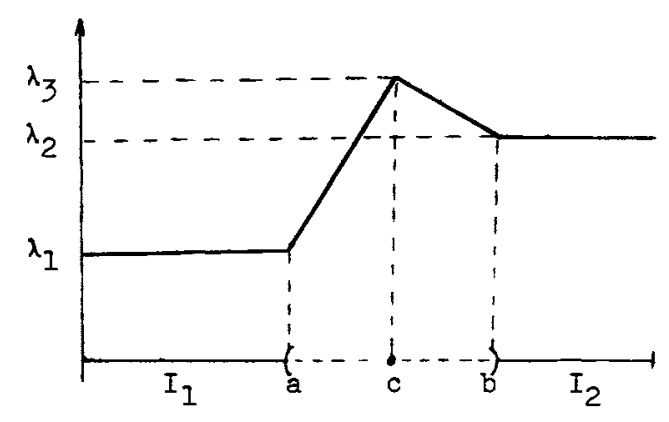


where $\lambda_{3}$ is such that

$$
\begin{aligned}
\int_{a}^{b} & \tilde{\psi}^{\prime}(t) d t=\psi(b)-\psi(a) \\
\Rightarrow & \left(\frac{b-a}{2}\right)\left(\frac{\lambda_{1}+\lambda_{2}}{2}\right)+\left(\frac{b-a}{2}\right)\left(\frac{\lambda_{2}+\lambda_{3}}{2}\right) \\
\quad & =(b-a) \psi^{\prime}(\xi), \quad \exists \xi \in(a, b) \\
\Rightarrow & \lambda_{3}=2 \psi^{\prime}(\xi)-\frac{\lambda_{1}+\lambda_{2}}{2}=2 \psi^{\prime}(\xi)-\frac{\psi^{\prime}\left(c_{1}\right)+\psi^{\prime}\left(c_{2}\right)}{2} .
\end{aligned}
$$

Therefore, $\tilde{\psi}^{\prime}$ satisfies a condition of Hölder with the same $\varepsilon$ and constant $\tilde{C}=2(A+2) C$, and if we exchange $\varepsilon$ for an $\tilde{\varepsilon}$ a little smaller, we have proved the nearness between $\tilde{\psi}$ and $\psi$ in the $C^{1+\varepsilon}$ topology.

Given the lemma, the result follows from the following observations:

i) the set of ratios $\left\{\left|U_{2}\right| /\left|U_{1}\right| \mid U_{1}, U_{2}\right.$ are gaps of $K$ such that there is no gap $V$ of $K$ with $|V|>2 \max \left\{\left|U_{1}\right|,\left|U_{2}\right|\right\}$ between $U_{1}$ and $U_{2}$ and $\left.\frac{1}{2}<\left|U_{2}\right| /\left|U_{1}\right|<2\right\}$ is finite when $K$ is generalized affine, being equal to the set of ratios between the gaps of the first steps of the construction of $K$ that are between $1 / 2$ and 2 ;

ii) given a finite number of gaps in a generalized affine Cantor set it is possible to modify a little the initial partition that defines the set by keeping it generalized affine of the same type so that the ratio two of these gaps never be equal to 1 .

Since for generalized affine Cantor sets the distortion starting from a certain stage of the construction is null, it is enough to make the ratio between two gaps crated up to this step (a finite number) never equal to 1 , so that $\left(^{*}\right)$ be satisfied, and we have a point of continuity of lateral thicknesses.

Observe that the affine Cantor sets of type

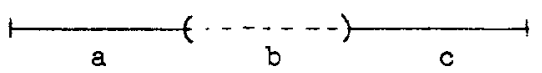

are such that the ratio between the lenght of a gap and the lenghts of the nearest gaps larger than it are always far from 1 , and are therefore points of continuity of the lateral thicknesses, as we have stated. 


\section{EXAMPLES ABOUT ARITHMETIC DIFFERENCES AND STABLE INTERSECTIONS}

Example III.1. - Let us consider the sets

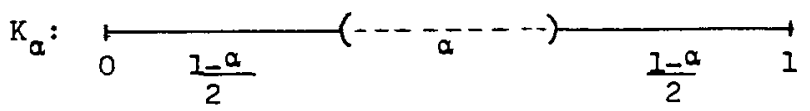

The Hausdorff dimmension of $K_{\alpha}$ is equal to: $-\frac{\log 2}{\log \left(\frac{1-\alpha}{2}\right)} \quad$ (see [PT2]), and $\tau\left(K_{\alpha}\right)=\tau_{L}\left(K_{\alpha}\right)=\tau_{R}\left(K_{\alpha}\right)=\frac{1-\alpha}{2 \alpha}$. If $\alpha>\frac{1}{2}$ we shall have $H D\left(K_{\alpha}\right)<\frac{1}{2}$, and $\left(K_{\alpha}-\lambda K_{\alpha}\right)$ will have measure zero for every $\lambda \in \mathbf{R}$.

If $\alpha<\frac{1}{3}, \tau\left(K_{\alpha}\right)>1$ and $\left(K_{\alpha}, \lambda K_{\alpha}\right)$ will have stable intersection, therefore $K_{\alpha}-\lambda K_{\alpha}$ will contain an interval, $\forall \lambda \in \mathbf{R}^{*}$.

We shall prove hereby that, when $\frac{1}{3}<\alpha<\frac{1}{2}, K_{\alpha}-K_{\alpha}$ has measure zero, but there are be intervals of values of $\lambda$ (that will depende on $\alpha$ ) for which $K_{\alpha}-\lambda K_{\alpha}$ contains an interval.

Proof. - Consider the set $K=K_{\alpha} \times K_{\alpha} \subset \mathbf{R}^{2} \cdot \mathbf{K}_{\alpha}-\mathbf{K}_{\alpha}$ is its projection over $\mathbf{R} \times\{0\}$ at an angle of $45^{\circ}$ (Fig. 1).
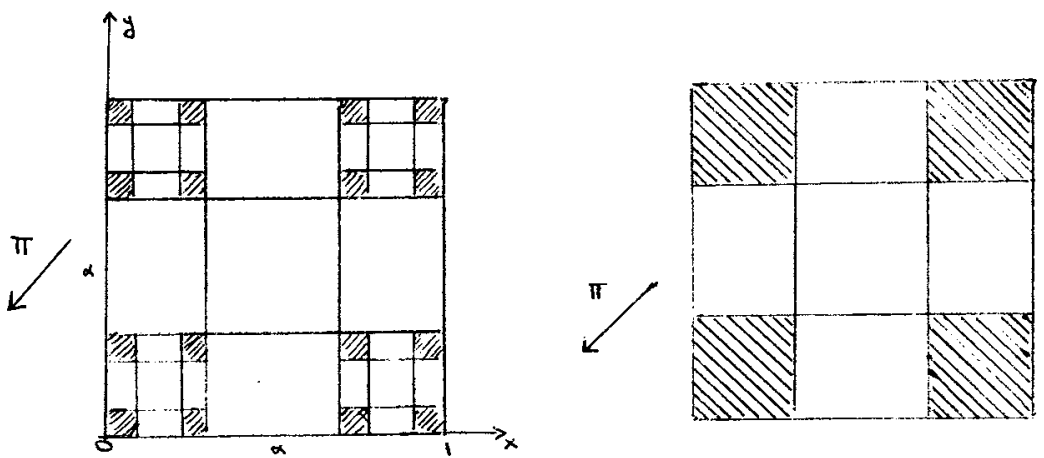

At the first step of the construction of $K_{\alpha} \times K_{\alpha}$, four congruent squares remain and the lower left square projects itself exactly over the one at the right higher corner (Fig. 2).

Therefore, by the self-similarity of $K_{\alpha}$, the construction continues as if they were one only square. In case $\alpha>\frac{1}{2}$, the projection of the first stage of the construction is not all the interval $[0,1]$, but the set $[-1,-\alpha] \cup\left[\frac{\alpha-1}{2}, \frac{1-\alpha}{2}\right] \cup[\alpha, 1]$ consisting of three connected components. 
From then on, the difference is constructed as an affine Cantor set, being equal to the set

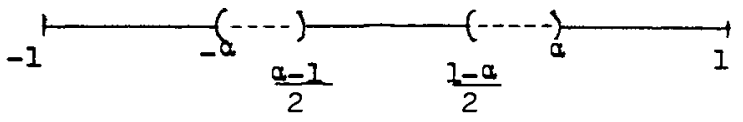

which has measure zero (and even Hausdorff dimension less than one).

The usual Cantor set $K_{\frac{1}{3}}$ can be approximated, on one hand, by sets $K_{\frac{1}{3}-\varepsilon}, \varepsilon>0$ that have stable self-intersection (and even $\left(K_{\frac{1}{3}-\varepsilon}, K_{\frac{1}{3}-\varepsilon}+1\right.$ has extremal stable intersection) and, on the other, by $K_{\frac{1}{3}+\varepsilon}, \varepsilon>0$, that has arithmetic self-difference with measure zero and Hausdorff dimension $<1$.

If the projection of the first step of the construction of $K_{\alpha} \times\left(\lambda K_{\alpha}\right)$ is surjective over the interval $[-\lambda, 1]$, the projections of all the stages will be surjective by self-similarity, and we shall have $K_{\alpha}-\lambda K_{\alpha}=[-\lambda, 1]$. We shall determine when this happens:

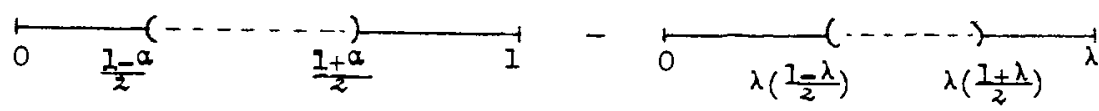

$=\left[-\lambda, \frac{1-\alpha}{2}-\lambda\left(\frac{1+\alpha}{2}\right)\right] \cup\left[\frac{1+\alpha}{2}-\lambda, 1-\lambda\left(\frac{1+\alpha}{2}\right)\right] \cup\left[-\lambda\left(\frac{1-\alpha}{2}\right), \frac{1-\alpha}{2}\right] \cup\left[\frac{1+\alpha}{2}-\right.$ $\left.\lambda\left(\frac{1-\alpha}{2}\right), 1\right]$, which is equal to the interval $[-\lambda, 1]$ if $\left(\frac{1-\alpha}{2}-\lambda\left(\frac{1+\alpha}{2}\right) \geq\right.$ $\frac{1+\alpha}{2}-\lambda, 1-\lambda\left(\frac{1+\alpha}{2}\right) \geq-\lambda\left(\frac{1-\alpha}{2}\right)$ and $\left.\frac{1-\alpha}{2} \geq \frac{1+\alpha}{2}-\lambda\left(\frac{1-\alpha}{2}\right)\right)$ or $\left(\frac{1-\alpha}{2}-\lambda\left(\frac{1+\alpha}{2}\right) \geq-\lambda\left(\frac{1-\alpha}{2}\right), \frac{1-\alpha}{2} \geq \frac{1+\alpha}{2}-\lambda\right.$ and $1-\lambda\left(\frac{1+\alpha}{2}\right) \geq$ $\left.\frac{1+\alpha}{2}-\lambda\left(\frac{1-\alpha}{2}\right)\right)$, that is, if $\left(\lambda \geq \frac{2 \alpha}{1-\alpha}\right.$ and $\left.\lambda \leq 1\right)$ or $\left(\lambda \leq \frac{2 \alpha}{1-\alpha} e \lambda \geq \alpha\right)$.

As $\alpha>\frac{1}{3}, \frac{2 \alpha}{1-\alpha}>1$ and the first possibility never occurs. However, as $\alpha<1 / 2$, the interval $\left[\alpha, \frac{1-\alpha}{2 \alpha}\right]$ is nonempty, and it is an interval of values of $\lambda$ for which $K_{\alpha}-\lambda K_{\alpha}=[-\lambda, 1]$. For self-similarity, if $\lambda \in \cup_{k=0}^{\infty}\left[\left(\frac{1-\alpha}{2}\right)^{k} \alpha,\left(\frac{1-\alpha}{2}\right)^{k} \cdot \frac{1-\alpha}{2 \alpha}\right], K_{\alpha}-\lambda K_{\alpha}$ will contain an interval.

An interesting observation is that the second part of this example is not as rigid as it looks. In fact, if $\lambda \in\left(\alpha, \frac{1-\alpha}{2 \alpha}\right), \frac{1}{3}<\alpha<\frac{1}{2}$ and $t \in(-\lambda, 1)$ then $K_{\alpha}$ has an intersection with $\lambda K_{\alpha}+t$ that we cannot prove to be stable, and $\left(K_{\alpha}, \lambda K_{\alpha}+1\right)$ and $\left(\lambda K_{\alpha}-\lambda, K_{\alpha}\right)$ have no extremal stable intersections, as we shall prove in the next sections. However, these intersections cannot be destroyed by applying diffeomorphisms $\varphi$ and $\psi C^{1}$-near the identity to $K_{\alpha}$ and $\lambda K_{\alpha}+t$. More precisely, given $t \in(-\lambda, 1)$ there exists $\delta>0$ such 
that if $\|\varphi-I d\|_{1}<\delta$ and $\|\psi-I d\|_{1}<\delta$ then $\varphi\left(K_{\alpha}\right) \cap \psi\left(\lambda K_{\alpha}+t\right) \neq \emptyset$. Besides, $\left(K_{\alpha}, \lambda K_{\alpha}+1\right)$ and $\left(\lambda K_{\alpha}-\lambda, K_{\alpha}\right)$ have extremal intersections such that there exists $\delta>0$ so that if $\|\varphi-I d\|_{1}<\delta$ and $\|\psi-I d\|_{1}<\delta$ then $\varphi\left(K_{\alpha}\right) \cap \psi\left(\lambda K_{\alpha}+1\right) \neq \emptyset$ and $\varphi\left(K_{\alpha}\right) \cap \psi\left(\lambda K_{\alpha}-\lambda\right) \neq \emptyset$, provided that their support intervals have nonempty intersections.

In fact, the ratio between the lenghts of a gap of $\lambda K_{\alpha}$ and a gap of $K_{\alpha}$ is equal to $\lambda\left(\frac{1-\alpha}{2}\right)^{k}$, for some $k \in \mathbf{Z}$. If $\lambda \in\left(\alpha, \frac{1-\alpha}{2 \alpha}\right)$ and $\delta$ is small enough then the ratio between a gap of $\varphi\left(K_{\alpha}\right)$ and a gap of $\psi\left(\lambda K_{\alpha}\right)$ will be larger than $\frac{2 \alpha}{1-\alpha}$ or smaller than $\frac{1-\alpha}{2 \alpha}$, for all the $\varphi, \psi \in C^{1}$ with $\|\varphi-I d\|_{1}<\delta$ and $\|\psi-I d\|_{1}<\delta$. Therefore, as in the proof of "gap lemma", if we have linked gap pair $(U, \tilde{U})$, for instance as below

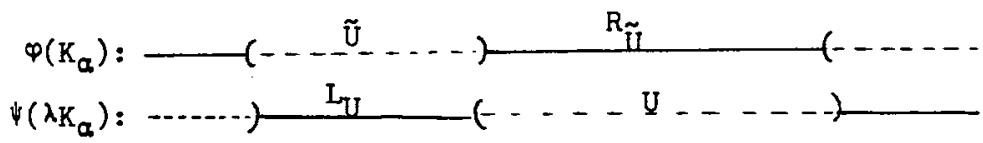

where we suppose $U>\tilde{U} \Rightarrow U>\frac{2 \alpha}{1-\alpha} \tilde{U}$, but we have $\tau_{L}\left(\psi\left(\lambda K_{\alpha}\right)\right) \simeq$ $\tau_{L}\left(K_{\alpha}\right)=\frac{1-\alpha}{2 \alpha} \Rightarrow|L|>|\tilde{U}| \Rightarrow$ we obtain a smaller gap pair. We proceed as in the proof of "gap lemma" and obtain the desired result (that is, if $\varphi\left(K_{\alpha}\right)$ and $\psi\left(\lambda K_{\alpha}+t\right)$ have a linked gap pair therefore they have non-empty intersection).

Example III.2. - We shall show here examples of afinne Cantor sets whose Hausdorff dimension is very close to 1 and whose arithmethic self-difference has measure zero.

Let $\bar{K}_{k}=$

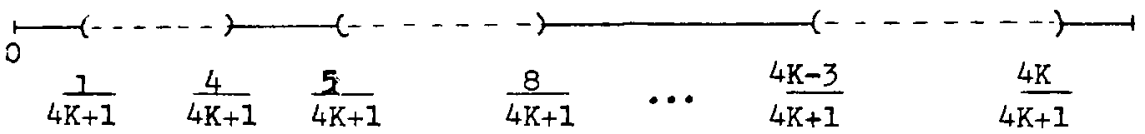

The projection of the first step of the construction of $\bar{K}_{k} \times \bar{K}_{k}$ is $\cup_{j=-k}^{k}\left[\frac{4 j-1}{4 k+1}, \frac{4 j+1}{4 k+1}\right]$, which is not all the interval $[-1,1]$ and by the same argument of self-similitude applied to $K_{\alpha}-K_{\alpha}, \bar{K}_{k}-\bar{K}_{k}$ will have measure zero (and even Hausdorff dimmension $<1$ : it will be an affine Cantor set) for the first step of the construction of $\bar{K}_{k} \times \bar{K}_{k}$ will consist of several congruent squares such that the projection of two of them either coincide or have empty intersection. 
Another way of seeing this is to observe that $\bar{K}_{k}$ is the set of numbers from $[0,1]$ in whose representation in base $4 k+1$ only the numbers $0,4,8, \ldots, 4 k$ appear $\Rightarrow \bar{K}_{k}-\bar{K}_{k}=\bar{K}_{k}+\bar{K}_{k}-1$, where $\bar{K}_{k}+\bar{K}_{k}$ is equal to affiine Cantor set $\{x \in[0,2]$ all digits of $x / 2$ in base $4 k+1$ are even\}.

The Hausdorff dimension of $\bar{K}_{k}$ is $\frac{\log (k+1)}{\log (4 k+1)}$ (it is the only $\lambda$ such that $(k+1) \cdot\left(\frac{1}{4 k+1}\right)^{\lambda}=1$; see [PT2] $)$.

Therefore, as $\lim _{k \rightarrow \infty} \frac{\log (k+1)}{\log (4 k+1)}=1$, we obtain the desired examples.

Example III.3. - Let $K=\left\{\sum_{i=1}^{\infty} \frac{\delta_{i}}{9^{2}} ; \delta_{i} \in\{0,4,6,8\}\right\} . K$ is an affine Cantor set. We have:

$$
\begin{gathered}
K+K=\{x+y \mid x \in K, y \in K\} \\
=\left\{\sum_{i=1}^{\infty} \frac{\mu_{i}}{9^{i}}, \mu_{i} \in\{0,4,6,8\}+\{0,4,6,8\}=\{0,4,6,8,10,12,14,16\}\right\} \\
K-K=\{x-y \mid x \in K, y \in K\} \\
=\left\{\sum_{i=1}^{\infty} \frac{\lambda_{i}}{9^{i}}, \lambda_{i} \in\{0,4,6,8\}-\{0,4,6,8\}=\{-8,-6,-4,-2,0,2,4,6,8\}\right\} .
\end{gathered}
$$

Therefore, $K+K=\left\{x \in \mathbf{R} \mid \frac{x}{2}=\sum_{i=1}^{\infty} \frac{\tilde{\mu}_{i}}{9^{i}}, \tilde{\mu}_{i} \in\{0,2,3,4,5,6,7,9\}\right\}$, which is an affine Cantor set, of measure zero and Hausdorff dimension smaller than on, while

$$
\begin{aligned}
K-K & =\left\{x \in \mathbf{R} \mid \frac{x+1}{2}=\sum_{i=1}^{\infty} \frac{\tilde{\lambda}_{i}}{9^{i}}, \tilde{\lambda}_{i} \in\{0,1,2,3,4,5,6,7,8\}\right\} \\
& =[-1,1] .
\end{aligned}
$$

Thus,

$$
K+K=\left\{x \in R \mid \frac{x}{2}=\sum_{i=0}^{\infty} \frac{\tilde{\mu}_{i}}{9^{i}}, \tilde{\mu}_{i} \in\{0,2,3,4,5,6,7,9\}\right\},
$$


which is an affine Cantor set, with measure zero and Hausdorff dimension less than 1 , while

$K-K=\left\{x \in \mathbf{R} \mid \frac{x+1}{2}=\sum_{i=1}^{\infty} \frac{\tilde{\lambda}_{i}}{9^{i}}, \tilde{\lambda}_{i} \in\{0,1,2,3,4,5,6,7,8\}\right\}=[-1,1]$.

Therefore, $K+K$ has measure zero, and $K-K$ is an interval.

Example III.4. - Let us consider the examples of Sannami [S] and of Bamón-Plaza-Vera [BPV], of dynamically define Cantor sets whose arithmetic self-difference is a Cantor set of positive measure. In these examples, the Cantor sets are central: a sequence $\underline{\alpha}=\left(\alpha_{1}, \alpha_{2}, \alpha_{3}, \ldots\right)$ of real numbers is given (in these cases we have $\lim _{i \rightarrow \infty} \alpha_{i}=\frac{1}{3}$ and $\alpha_{i}>\frac{1}{3}, \forall i$ ) and the central Cantor set $K_{\alpha}$ is defined in the following manner: we take the interval $[0,1]$, make a gap with the proportion $\alpha_{1}$, at the center of the interval, and at the first step two connected components are left out. At the $k$-th step $2^{k}$ connected components will be left out, and gaps of proportion $\alpha_{k}$ in each connected component resulting from step $k-1$ will be made. The intersection of the sets resulting from each step is the set $K_{\underline{\alpha}}$. The differentiability of $K_{\underline{\alpha}}$ is related to the speed of convergence of sequence $\left(\alpha_{i}\right)$. We can aproximate $\underline{\alpha}$ by $\underline{\alpha}+\varepsilon:=\left(\alpha_{i}+\varepsilon\right)_{i \in \mathrm{N}}$, obtaining sets $K_{\underline{\alpha}+\varepsilon}$ as diferentiable as $K_{\alpha}$. From the calculation of the measure of the arithmetic self-difference of central Cantor sets (which is made in the referred articles) we can deduce that, if $\varepsilon>0$ then $\lambda\left(K_{\underline{\alpha}+\varepsilon}-K_{\underline{\alpha}+\varepsilon}\right)=0$. On the other hand, if $\varepsilon<0$ we have $\tau_{\text {loc }}\left(K_{\underline{\alpha}+\varepsilon}\right)>1$, and thus $\left(K_{\underline{\alpha}+\varepsilon}, K_{\underline{\alpha}+\varepsilon}\right)$ will have stable intersection, and $\left(K_{\underline{\alpha}+\varepsilon}, K_{\underline{\alpha}+\varepsilon}+1\right)$ will have extremal stable intersection. In our last considerations we are referring to the sequence $\underline{\alpha}$ that appear in the mentioned examples (for which $\lim _{i \rightarrow \infty} \alpha_{i}=\frac{1}{3}$ ). In this cases we have $\tau_{\text {loc }}\left(K_{\underline{\alpha}}\right)=1$, as we observed in the comments after the proof of Proposition II.1.

\section{CRITERIA FOR STABLE AND EXTREMAL STABLE INTERSECTIONS AND GENERALIZED THICKNESS TEST}

We have seen in the preceding sections some examples of stable and extremal stable intersections of dynamically defined Cantor sets. In these examples the product of the right thickness of each of these sets by the left thickness of the other was always greater than one, so that it was possible to apply Proposition II.1. 
In this section we shall give examples of stable and extremal stable intersections of Cantor sets with small lateral thicknesses, and we shall give more general criteria for stable and extremal stable intersections that generalize the idea of thickness. In the case of affine Cantor sets, it is possible to test these criteria by a finite algorithm.

We shall also prove a theorem that characterizes when an arithmetic difference $K_{2}-K_{1}$, of two dynamically defined Cantor sets, such that the right extreme of $K_{1}$ coincides with the left one of $K_{2}$, contains an interval of the type $[0, \varepsilon], \varepsilon>0$. This theorem will give us several examples of pairs of Cantor sets $\left(K_{1}, K_{2}\right)$ which do not have extremal stable intersection.

Example IV.1. - Consider the affine Cantor sets

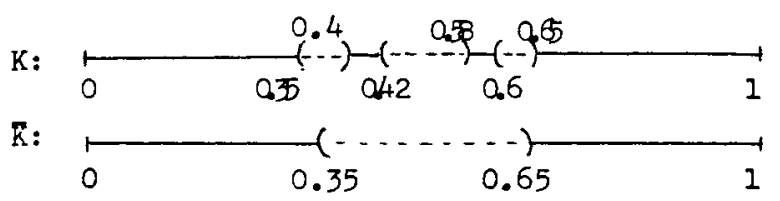

We have $\tau(\bar{K})=7 / 6>1$, and thus $\bar{K}$ intersects itself stably. Observe that $K$ contains $\bar{K}$ (we simply forget the two central intervals of the Markov partition of $K$, and consider the Cantor set defined by $\left.\psi\right|_{K_{1}}$ and $\left.\psi\right|_{K_{4}}$, which coincides with $\bar{K}$ ).

For the same reason, a Cantor set $K_{1}$ near $K$ contains a Cantor set $\bar{K}_{1}$ near $\bar{K}$. This implies that $(K, K)$ have stable intersection, but $\tau_{R}(K)=\tau_{L}(K)=\frac{2}{5}<1$. This example shows that the lateral thickness test of Proposition II.1 does not characterize stable intersections, and also shows that we can have $\bar{K} \subset K$ with $\tau(\bar{K})>\tau(K)$ (same for $\tau_{R}$ and $\tau_{L}$ ).

We shall see now a criteria for stable and extremal stable intersections which we will study first for affine Cantor sets, and later for the general cases.

Let us consider the affine Cantor sets

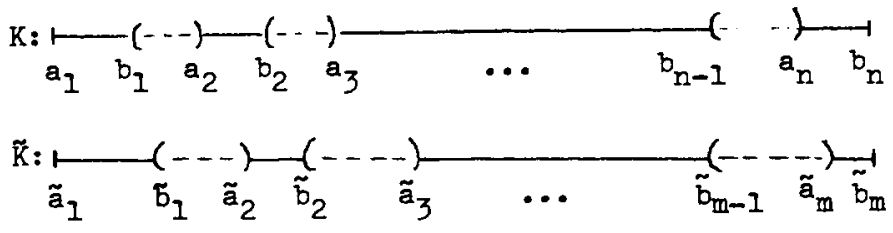

With Markov domains respectively $P=\cup_{j=1}^{n}\left[a_{j}, b_{j}\right]$ and $\tilde{P}=\cup_{j=1}^{m}\left[\tilde{a}_{j}, \tilde{b}_{j}\right]$. We say that $(K, \tilde{K})$ (or $(P, \tilde{P}))$ satisfies the Generalized Thickness Test 
(GTT) if for every $t \in \mathbf{R}$ and every $\lambda \in \mathbf{R}_{+}^{*}$ either $\lambda P+t$ is contained in a gap of $\tilde{P}$ or $\tilde{P}$ is contained in a gap of $\lambda P+t$ or $(\lambda P+t) \cap \tilde{P} \neq \emptyset$.

This is equivalent to the condition that the arithmetic difference $\tilde{P}-\lambda P$ contains the set of the $t \in \mathbf{R}$ such that, neither $\tilde{P}$ is contained in a gap of $\lambda P+t$ nor $\lambda P+t$ is contained in a gap of $\tilde{P}$, for every $\lambda \in \mathbf{R}_{+}^{*}$.

Geometrically, this means that every line that intercepts $\left[a_{1}, b_{n}\right] \times\left[\tilde{a}_{1}, \tilde{b}_{m}\right]$ and makes an angle between 0 and $\pi / 2$ with ther horizontal axis has an intersection with $\left[a_{1}, b_{n}\right] \times\left[\tilde{a}_{1}, b_{m}\right]$, which is either contained in a vertical strip $\left(b_{i}, a_{i+1}\right) \times\left[\tilde{a}_{1}, \tilde{b}_{m}\right]$ or in an horizontal strip $\left[a_{1}, b_{n}\right] \times\left(\tilde{b}_{i}, \tilde{a}_{1+1}\right)$, or then intercepts $P \times \tilde{P}$.

Algebraically, the set of the $t$ such that $\lambda P+t$ is contained in some $\operatorname{gap}\left(\tilde{b}_{i}, \tilde{a}_{i+1}\right)$ of $\tilde{P}$ is $\cup_{i=1}^{m-1}\left\{t \in \mathbf{R} \mid\left[\lambda a_{1}+t, \lambda b_{n}+t\right] \subset\left(\tilde{b}_{i}, \tilde{a}_{i+1}\right)\right\}=$ $\cup_{i=1}^{m-1}\left(\tilde{b}_{i}-\lambda a_{1}, \tilde{a}_{i+1}-\lambda b_{n}\right)$, and the set of the $t$ such that $\tilde{P}$ is contained in some gap $\left(\lambda b_{i}+t, \lambda a_{i+1}+t\right)$ of $\lambda P+t$ is $\cup_{i=1}^{n-1}\left(\tilde{b}_{m}-\lambda a_{i+1}, \tilde{a}_{1}-\lambda b_{i}\right)$. Since the arithmetic difference $\tilde{P}-\lambda P$ is equal to

$$
\bigcup_{\substack{1 \leq i \leq m \\ 1 \leq j \leq n}}\left[\tilde{a}_{i}-\lambda b_{j}, \tilde{b}_{i}-\lambda a_{j}\right]
$$

we have that $(K, \tilde{K})$ satisfies the GTT $\Leftrightarrow$ for any $\lambda \in \mathbf{R}_{+}^{*}$,

$$
\bigcup_{\substack{1 \leq i \leq m \\ 1 \leq j \leq n}}\left[\tilde{a}_{i}-\lambda b_{j}, \tilde{b}_{i}-\lambda a_{j}\right] \supset\left[\tilde{a}_{1}-\lambda b_{n}, \tilde{b}_{m}-\lambda a_{1}\right] \backslash(A \cup B),
$$

where

$$
A=\bigcup_{i=1}^{m-1}\left(\tilde{b}_{i}-\lambda a_{1}, \tilde{a}_{i+1}-\lambda b_{n}\right) \quad \text { and } \quad B=\bigcup_{i=1}^{n-1}\left(\tilde{b}_{m}-\lambda a_{i+1}, \tilde{a}_{1}-\lambda b_{i}\right) .
$$

Note that we have a finite number of possibilities for the relative position of the intervals $\left[\tilde{a}_{i}-\lambda b_{j}, \tilde{b}_{i}-\lambda a_{j}\right],\left(\tilde{b}_{i}-\lambda a_{1}, \tilde{a}_{i+1}-\lambda b_{n}\right)$ and $\left(\tilde{b}_{m}-\lambda a_{i+1}, \tilde{a}_{1}-\lambda b_{i}\right)$, and to each possibility there corresponds a set of values of $\lambda$ determined by a finite number of inequalities of the type $A-B \lambda \leq C-D \lambda$, or $A-B \lambda<C-D \lambda$, which determine a real interval of values of $\lambda$ whose extremes are functions of the $a_{i}, \tilde{a}_{j}, b_{i}$ and $\tilde{b}_{j}$. The GTT means that for each relative position of the intervals such that $\left(^{*}\right)$ does not hold we should have the corresponding interval of values of $\lambda$ empty, which can be translated in a finite number of inequalities involving the $a_{i}, \tilde{a}_{j}, b_{i}$ and $\tilde{b}_{j}$. If these inequalities are strict, we say that $(K, \tilde{K})$ satisfies the GTT strictly. 
If $(K, \tilde{K})$ satisfies the GTT, then $(\lambda K+t, \tilde{K})$ also satisfies the GTT, $\forall t \in \mathbf{R}, \forall \lambda \in \mathbf{R}_{+}^{*}$. Therefore, if $(K, \tilde{K})$ satisfies the GTT, $I$ is an interval of the $i^{\text {th }}$ step of the construction of $K$ (that is, $I$ is a connected component of $\left.\psi^{1-i}(P)\right)$ and $J$ is an interval of the $j^{\text {th }}$ step of the construction of $\tilde{K}$ then $(K \cap I, \tilde{K} \cap J)$ satisfies the GTT. If $(U, \tilde{U})$ is a pair of linked gaps, $U$ being a gap of the $i$ th step of the construction of $K\left(\psi^{i}(U)=U^{\prime}, U^{\prime}\right.$ gap of $P$ ) contained in some interval $I$ of the $\mathrm{i}^{\text {th }}$ step, and $\tilde{U}$ being a gap of the $j^{\text {th }}$ step of the construction of $\tilde{K}$, contained in some interval $J$, then, applying the GTT to $(K \cap I, \tilde{K} \cap J)$, we will obtain a gap pair $\left(U_{1}, \tilde{U}_{1}\right)$, where $U_{1}$ is a gap of the $i^{\text {th }}$ step of the construction of $K$ and $\tilde{U}_{1}$ is a gap of $\tilde{K}$ of a step superior to the $j^{\text {th }}$, or a gap pair $\left(U_{1}, \tilde{U}_{1}\right)$ where $U_{1}$ is a gap of $K$ of a step superior to the $i$ th and $\tilde{U}_{1}$ is a gap of $\tilde{K}$ of the $j$ th step. (See the figure below). Consequently, if $(K, \tilde{K})$ satisfies the GTT, then either $K$ is contained in a gap of $\tilde{K}$, or $\tilde{K}$ is contained in a gap of $K$ or $K \cap \tilde{K} \neq \emptyset$, as in Proposition II.1.
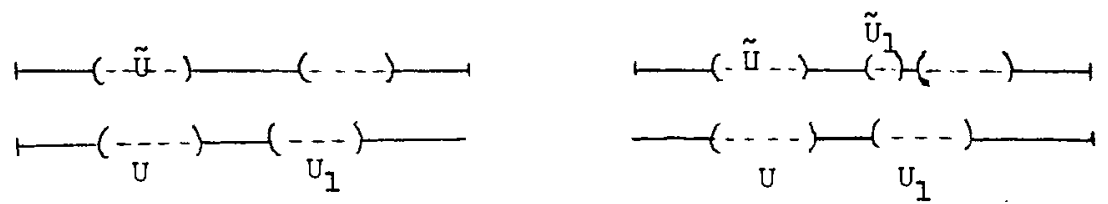

If $(K, \tilde{K})$ satisfies the GTT strictly and there is a pair of linked gaps of $K$ and $\tilde{K}$, then $K$ intersects $\tilde{K}$ stably, and if the right extreme of $K$ coincides with the left extreme of $\tilde{K}$, then $(K, \tilde{K})$ has extremal stable intersection.

Note that the conditions of the GTT are closed, while those of the Strict GTT are open.

To apply the GTT to generalized affine Cantor sets $K$ and $\tilde{K}$ defined by expansive functions $\psi$ and $\tilde{\psi}$, and with Markov partitions $\left\{K_{1}, K_{2}, \ldots, K_{n}\right\}$ and $\left\{\tilde{K}_{1}, \tilde{K}_{2}, \ldots, \tilde{K}_{m}\right\}$, we must apply GTT to any pair $\left(\psi\left(K_{i}\right) \cap P, \tilde{\psi}\left(\tilde{K}_{j}\right) \cap \tilde{P}\right)$, where $P$ and $\tilde{P}$ are the Markov domains of $K$ and $\tilde{K}$.

We shall see later how to apply the GTT when $K$ and $\tilde{K}$ are not affine, and when some $\psi_{i}$ or some $\tilde{\psi}_{i}$ is decreasing.

Let us see now to apply the GTT when $m=n=2$ : Let
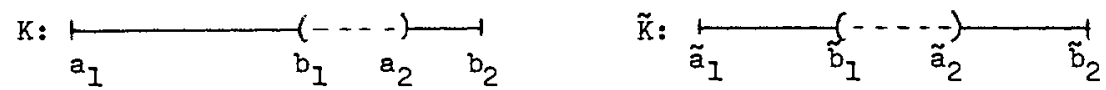

Let $I_{1}:=\left[\tilde{a}_{1}-\lambda b_{2}, \tilde{b}_{1}-\lambda a_{2}\right], I_{2}:=\left[\tilde{a}_{1}-\lambda b_{1}, \tilde{b}_{1}-\lambda a_{1}\right], I_{3}:=\left[\tilde{a}_{2}-\right.$ $\left.\lambda b_{2}, \tilde{b}_{2}-\lambda a_{2}\right]$ and $I_{4}:=\left[\tilde{a}_{2}-\lambda b_{1}, \tilde{b}_{2}-\lambda a_{1}\right]$. 
Since $\left(\tilde{b}_{1}-\lambda a_{1}, \tilde{a}_{2}-\lambda b_{2}\right) \cup\left(\tilde{b}_{2}-\lambda a_{2}, \tilde{a}_{1}-\lambda b_{1}\right)=\operatorname{conv}\left(I_{2} \cup I_{3}\right) \backslash\left(I_{2} \cup I_{3}\right)$, where $\operatorname{conv}\left(I_{2} \cup I_{3}\right)$ is the convex hull of $I_{2} \cup I_{3}$, we will have $(*)$ satisfied if and only if $I_{1} \cap\left(I_{2} \cup I_{3}\right) \neq \emptyset$ and $I_{4} \cap\left(I_{2} \cup I_{3}\right) \neq \emptyset, \forall \lambda \in \mathbf{R}_{+}^{*}$ (note that $I_{1} \cap I_{4}=\emptyset$ is always empty).

We have:

$$
\begin{aligned}
& I_{1} \cap I_{2} \neq \emptyset \Leftrightarrow \tilde{b}_{1}-\tilde{a}_{1} \geq \lambda\left(a_{2}-b_{1}\right), I_{1} \cap I_{3} \neq \emptyset \Leftrightarrow \tilde{a}_{2}-\tilde{b}_{1} \leq \lambda\left(b_{2}-a_{2}\right) \\
& I_{4} \cap I_{2} \neq \emptyset \Leftrightarrow \tilde{a}_{2}-\tilde{b}_{1} \leq \lambda\left(b_{1}-a_{1}\right), I_{4} \cap I_{3} \neq \emptyset \Leftrightarrow \tilde{b}_{2}-\tilde{a}_{2} \geq \lambda\left(a_{2}-b_{1}\right)
\end{aligned}
$$

Thus,

$$
I_{1} \cap\left(I_{2} \cup I_{3}\right)=\emptyset \quad \Leftrightarrow \quad \frac{\tilde{b}_{1}-\tilde{a}_{1}}{a_{2}-b_{1}}<\lambda<\frac{\tilde{a}_{2}-\tilde{b}_{1}}{b_{2}-a_{2}}
$$

and

$$
I_{4} \cap\left(I_{2} \cup I_{3}\right)=\emptyset \quad \Leftrightarrow \quad \frac{\tilde{b}_{2}-\tilde{a}_{2}}{a_{2}-b_{1}}<\lambda<\frac{\tilde{a}_{2}-\tilde{b}_{1}}{b_{1}-a_{1}}
$$

The TEG will be satisfied $\Leftrightarrow$

$$
\frac{\tilde{a}_{2}-\tilde{b}_{1}}{b_{2}-a_{2}} \leq \frac{\tilde{b}_{1}-\tilde{a}_{1}}{a_{2}-b_{1}} \quad \text { e } \quad \frac{\tilde{a}_{2}-\tilde{b}_{1}}{b_{1}-a_{1}} \leq \frac{\tilde{b}_{2}-\tilde{a}_{2}}{a_{2}-b_{1}}
$$

that is, if and only if

$$
\begin{aligned}
& \frac{\tilde{b}_{1}-\tilde{a}_{1}}{\tilde{a}_{2}-\tilde{b}_{1}} \cdot \frac{b_{2}-a_{2}}{a_{2}-b_{1}} \geq 1 \quad \text { and } \quad \frac{\tilde{b}_{2}-\tilde{a}_{2}}{\tilde{a}_{2}-\tilde{b}_{1}} \cdot \frac{b_{1}-a_{1}}{a_{2}-b_{1}} \geq 1 \\
& \Leftrightarrow \quad \tau_{R}\left(K_{1}\right) \tau_{L}\left(K_{2}\right) \geq 1 \quad \text { and } \tau_{L}\left(K_{1}\right) \tau_{R}\left(K_{2}\right) \geq 1 .
\end{aligned}
$$

We also have that $\left(K_{1}, K_{2}\right)$ satisfies the GTT strictly if and only if $\tau_{R}\left(K_{1}\right) \tau_{L}\left(K_{2}\right)>1$ and $\tau_{L}\left(K_{1}\right) \tau_{R}\left(K_{2}\right)>1$.

We shall see now how to apply an adaptation of the GTT to dynamically defined Cantor sets, not necessarily affine. Observe first that applying the GTT to a pair of affine Cantor sets $\left(K_{1}, K_{2}\right)$ is equivalent to applying them to $\left(\lambda K_{1}, \mu K_{2}\right), \forall \lambda, \mu \in \mathbf{R}_{+}^{*}$. Consequently, we can suppose that the support intervals of $K_{1}$ and $K_{2}$ are equal to $[0,1]$. We shall have in this case the following normalized representations of $K_{1}$ and $K_{2}$ :

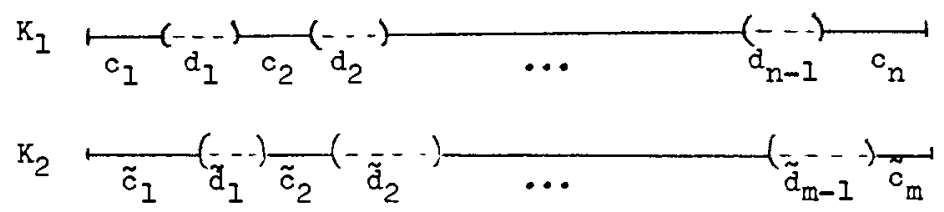


where $c_{1}+d_{1}+c_{2}+d_{2}+\ldots+d_{n-1}+c_{n}=1$ and $\tilde{c}_{1}+\tilde{d}_{1}+\tilde{c}_{2}+\tilde{d}_{2}+\ldots+\tilde{d}_{m-1}+$ $\tilde{c}_{m}=1$. If $\underline{c}:=\left(c_{1} c_{2}, \cdots, c_{n}\right), \underline{d}:=\left(d_{1}, \ldots, d_{n-1}\right), \underline{\tilde{c}}:=\left(\tilde{c}_{1}, \ldots, \tilde{c}_{m}\right)$ and $\underline{\tilde{d}}=\left(\tilde{d}_{1}, \ldots, \tilde{d}_{m-1}\right)$ then the conditions of the GTT applied to the pair $\left(K_{1}, K_{2}\right)$ can be translated in a finite number of inequalities $f_{i}(\underline{c}, \underline{d}, \underline{\tilde{c}}, \underline{\tilde{d}}) \geq 0$, for $1 \leq i \leq \ell$. As we saw before, if $I$ and $I_{2}$ are intervals of any steps of the constructions of $K_{1}$ and $K_{2}$ then applying the GTT to $\left(K_{1}, K_{2}\right)$ is equivalent of applying them to $\left(I_{1} \cap K_{1}, I_{2} \cap K_{2}\right)$.

For a pair of dynamically defined Cantor sets $\left(K_{1}, K_{2}\right)$ choose $I_{1}$ and $I_{2}$ intervals of some steps of the constructions of $K_{1}$ and $K_{2}$, and apply the GTT to $\left(\psi_{1}^{-n_{1}}\left(P_{1}\right) \cap I_{1}, \psi_{2}^{-n_{2}}\left(P_{2}\right) \cap I_{2}\right)$ (as for affine Cantor sets), where $P_{i}$ is the Markov domain of $K_{i}, \psi_{i}$ is the expansive function that defines $K_{i}$ and $n_{i}$ is the step of $I_{i}, i=1,2$. This test, after normalization, can be translated in inequalities $f_{i}\left(\underline{c}\left(P_{1}^{*}\right), \underline{d}\left(P_{1}^{*}\right), \underline{\tilde{c}}\left(P_{2}^{*}\right), \underline{\tilde{d}}\left(P_{2}^{*}\right)\right) \geq 0,1 \leq i \leq \ell$, where $P_{i}^{*}=\psi_{i}^{-n_{i}}\left(P_{i}\right) \cap I_{i}=1,2$, which do not depend on the choice of $I_{1}$ and $I_{2}$ if $K$ is affine. In the general case, we say that $\left(K_{1}, K_{2}\right)$ satisfies the GTT if

$$
A_{j}\left(K_{1}, K_{2}\right):=\inf _{I_{1}, I_{2}} f_{j}\left(\underline{c}\left(P_{1}^{*}\right), \underline{d}\left(P_{1}^{*}\right), \tilde{c}\left(P_{2}^{*}\right), \underline{\tilde{d}}\left(P_{2}^{*}\right)\right) \geq 0 \quad \text { for } 1 \leq j \leq \ell,
$$

and that $\left(K_{1}, K_{2}\right)$ satisfies the GTT strictly if $A_{j}\left(K_{1}, K_{2}\right)>0,1 \leq j \leq \ell$. Note that, as in the affine case, the strict GTT implies stable intersection and extremal stable intersection of $\left(K_{1}, K_{2}\right)$ if there is a gap pair or if the extremes coincide, respectively. Note also that $\ell$ depends only on the combinatorial type of $K_{1}$ and $K_{2}$. The first statement follows from the continuity of the $A_{j}\left(K_{1}, K_{2}\right)$ in the $C^{1+\varepsilon}$ topology, which follows from the bounded distortion property for dynamically defined Cantor sets (see the proof of the continuity of $\tau(K)$ in [PT2]). As for $\tau(K)$ we can define $\left(A_{j}\right)_{\mathrm{loc}}\left(K_{1}, K_{2}\right), 1 \leq j \leq \ell$ and if $\left(A_{j}\right)_{\mathrm{loc}}\left(K_{1}, K_{2}\right)>0,1 \leq j \leq \ell$ then $\left(K_{1}, K_{2}\right)$ have extremal stable intersection, provided that the extremes coincide.

Remark. - If any of the functions that define $K_{1}$ or $K_{2}$ is decreasing, then we must apply the GTT for $\left(\lambda P_{1}+t, P_{2}\right), \forall \lambda \in \mathbf{R}^{*}$, and not only for $\lambda$ positive, that is, it is necessary to make sure that either $\lambda P_{1}+t$ is contained in a gap of $P_{2}$ or $P_{2}$ is contained in a gap of $\lambda P_{1}+t$ or $\left(\lambda P_{1}+t\right) \cap P_{2} \neq \emptyset$, for every $t \in \mathbf{R}, \lambda \in \mathbf{R}^{*}$. This also applies to the dynamically defined case.

Example IV.2. - If

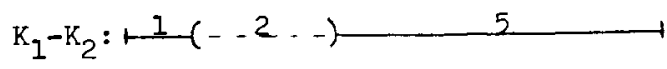


then $\left(K_{1}, K_{2}+8\right)$ have extremal stable intersection by the GTT. However, if $\tilde{K}_{1},=\tilde{K}_{2}$ is defined as below, by affine functions, then $\left(\tilde{K}_{1}, \tilde{K}_{2}+8\right)$ do not have extremal stable intersection. Inded, $\tilde{K}_{1}=\tilde{K}_{2}$ has gaps near the left extreme like:

and near the right extreme like

$$
\Rightarrow[-8,-8+\varepsilon] \not \subset \tilde{K}_{1}-\tilde{K}_{2} \forall \varepsilon>0 \text {. }
$$

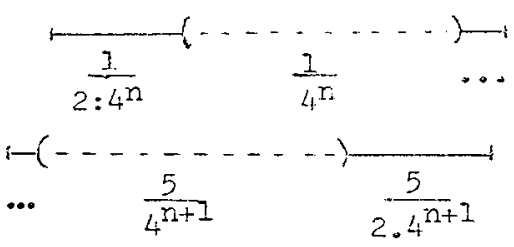

$\tilde{K}_{1}=\tilde{K}_{2}$

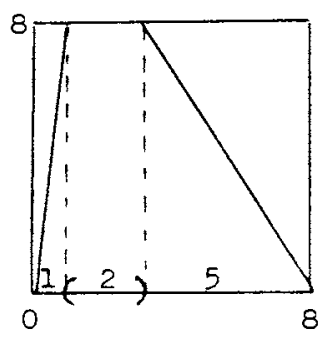

So, we see that it is really necessary to consider all the values of $\lambda \in \mathbf{R}^{*}$ if there is some decreasing function.

Example IV.3. - Let

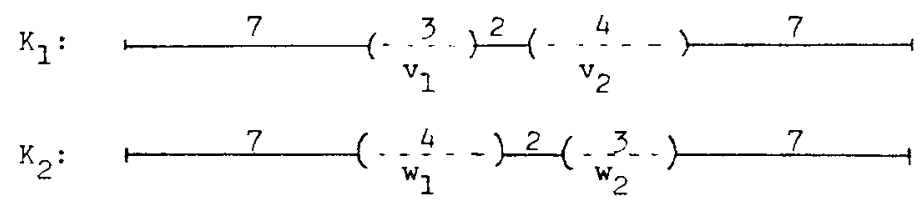

we have $\tau_{R}\left(K_{1}\right) \tau_{L}\left(K_{2}\right)=\frac{2}{3} \cdot \frac{2}{3}=\frac{4}{9}<1$, hence we cannot apply the lateral thicknesses test of Proposition II.1 to find stable intersections between $K_{1}$ and $K_{2}$. However, $\left(K_{1}, K_{2}\right)$ satisfies the GTT strictly. Indeed, if a gap $U$ of $K_{1}$ is of the type of $V_{1}$ (there exists $n$ such that $\psi^{n}(U)=V_{1}$, where $\psi$ is the function that defines $\left.K_{1}\right)$ then $\tau_{R}(U)=\frac{2}{3}$ and $\tau_{L}(U)=\frac{7}{3}$. If $\tilde{U}$ is a 
gap of $K_{2}$ of the kind of $W_{1}$ then $\tau_{R}(\tilde{U})=3$ and $\tau_{L}(\tilde{U})=\frac{7}{4}$. If $\tilde{U}$ is of the type of $W_{2}$ then $\tau_{R}(\tilde{U})=\frac{7}{3}$ and $\tau_{L}(\tilde{U})=\frac{2}{3}$.

Thus, a gap pair $(U, \tilde{U})$ satisfies the conditions of the gap lemma except if $U=: U_{0}^{1}$ is of the kind of $V_{1}$ and $\tilde{U}=: U_{0}^{2}$ is of the kind of $W_{2}$. In this case, we will have a situation like:

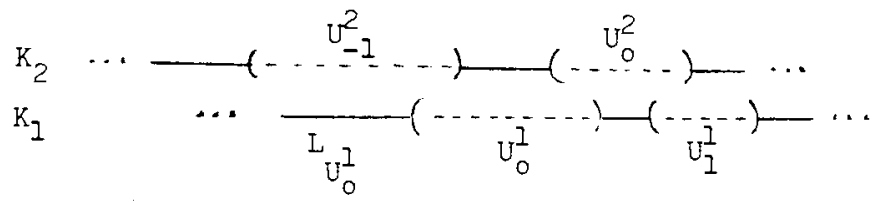

If $\left|U_{0}^{1}\right|>\left|L_{U_{0}^{2}}\right|$ then $\left|L_{U_{0}^{1}}\right|=\frac{7}{3}\left|U_{0}^{1}\right|>\left|U_{-1}^{2}\right|$, because $\left|U_{-1}^{2}\right|=2\left|L_{U_{0}^{2}}\right|$ and $2<\frac{7}{3}$. Thus, necessairly we will have an intersection between $\left(\lambda P_{1}+t\right)$ and $P_{2}$, and $\left(K_{1}, K_{2}\right)$ satisfies the GTT.

Example IV.4. - If $\alpha \in\left(\frac{1}{3}, \frac{1}{2}\right)$ then $\left(K_{\alpha}, K_{\alpha}\right)$ does not satisfy the GTT, the same ocurring in a neighbourhood of $\left(K_{\alpha}, K_{\alpha}\right)$. We will prove later that if $(K, \tilde{K})$ is close to $\left(K_{\alpha}, K_{\alpha}+1\right)$ then $(K, \tilde{K})$ will not have extremal stable intersection. Note that if $\alpha \in\left(\frac{1}{3}, \frac{1}{2}\right)$, then $H D\left(K_{\alpha}\right)>\frac{1}{2}$ and $H D\left(K_{\alpha}\right)+H D\left(K_{\alpha}\right)>1$ and thus extremal stable intersections are not dense in $\left\{\left(K_{1}, K_{2}\right) \mid H D\left(K_{1}\right)+H D\left(K_{2}\right)>1\right\}$.

Example IV.5. - Let us give now an example of a pair of Cantor sets $\left(K_{1}, K_{2}\right)$ which does not satisfy the GTT (and such that $\left(K_{1}, K_{2}+t\right)$ do not have extremal stable intersection, $\forall t \in \mathbf{R}$ ) such that if we substitute $K_{2}$ by an $\tilde{K}_{2}$ aparently "smaller", $\left(K, \tilde{K}_{2}\right)$ does satisfy the GTT. We will also exibit an $\tilde{K}_{1}$ such that $\left(\tilde{K}_{1}, \tilde{K}_{2}\right)$ does not satisfy the GTT (and does not have extremal stable intersection), but $\left(\tilde{K}_{1}, K_{2}\right)$ satisfies the GTT. This ilustrates the combinatorial character of the GTT.

Let
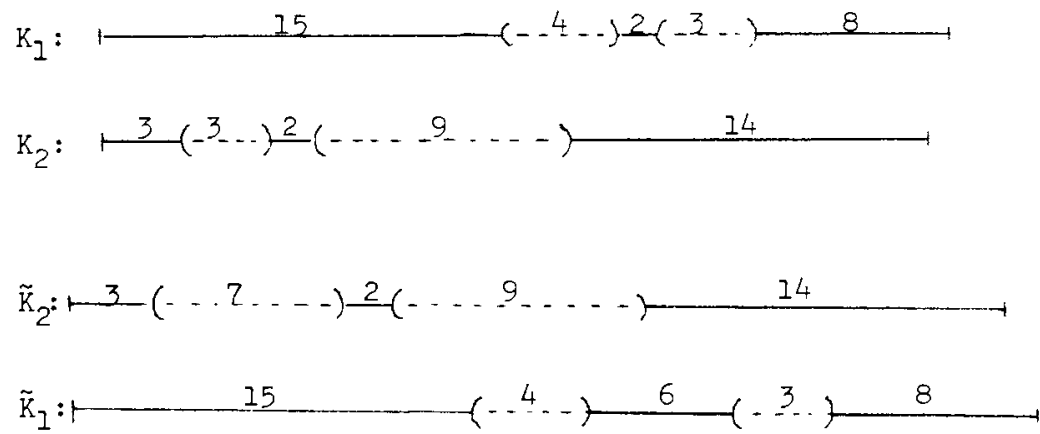
Surely $\left(K_{1}, K_{2}\right)$ and $\left(\tilde{K}_{1}, \tilde{K}_{2}\right)$ do not satisfy the GTT (and do not have extremal stable intersections, as we shall see later) by the pictures below:

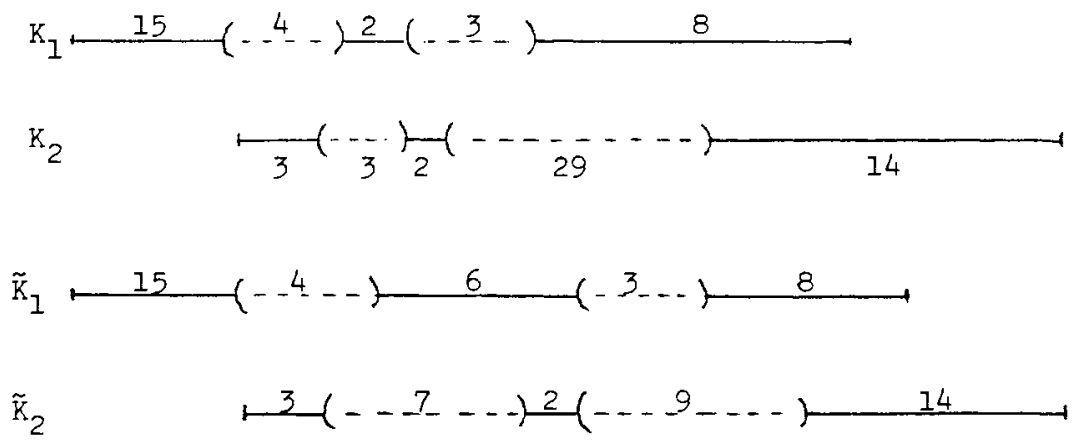

We have $\tau\left(\tilde{K}_{1}\right) \tau\left(K_{2}\right)=2 \cdot \frac{2}{3}=\frac{4}{3}>1$, and thus $\left(\tilde{K}_{1}, K_{2}\right)$ satisfies the GTT.

For $\left(P_{1}, \tilde{P}_{2}\right)$, the only pair of gaps that does not satisfy the condition of the gap lemma is $(U, V)$. In this case, we have:

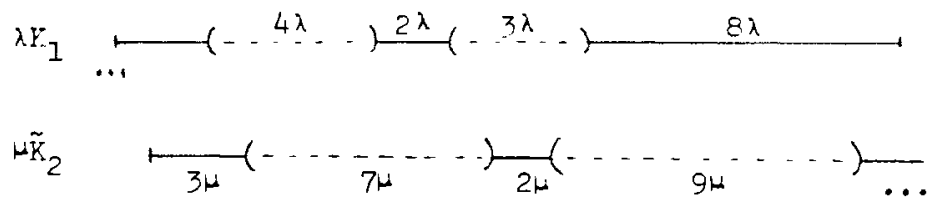

Also, to avoid intersections between $\lambda P_{1}+t$ and $\tilde{P}_{2}$ we must have $2+7+3<3 \lambda+2 \lambda+4 \lambda \Rightarrow 9<8 \lambda \Rightarrow$ the gap of $\tilde{P}_{2}$ of lenght 9 ends in the interval of $\lambda P_{1}+t$ of lenght $8 \lambda \Rightarrow\left(\lambda P_{1}+t\right) \cap \tilde{P}_{2} \neq \emptyset \Rightarrow\left(K_{1}, \tilde{K}_{2}\right)$ satisfies the GTT. Clearly, by the arguments above, $\left(\tilde{K}_{1}, K_{2}\right)$ and $\left(K_{1}, \tilde{K}_{2}\right)$ satisfy the GTT stably.

Example IV.6. - Let us give another proof of Theorem II.I.2, in which the sets $K_{1}$ and $K_{2}$ that we obtain are be simmetric, and can be seen as stable and unstable Cantor sets associated to a horseshoe in the sphere (in what follows, if $n_{0}=2^{k}, K_{1}$ and $K_{2}$ have the same combinatories as the $k^{\text {th }}$ power of the usual horseshoe). 
Let $h_{1}, h_{2}$ in the interval $(0,1)$ such that $h_{1}+h_{2}>1$, and let $a<h_{1}$ and $b<h_{2}$ such that $a+b>1$. Consider for each $\lambda \in(0,1)$, the affine Cantor set

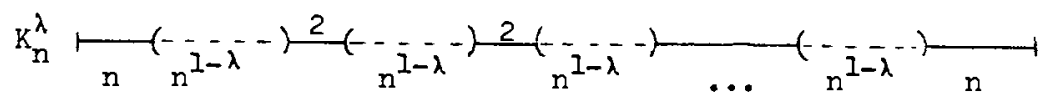

(with $\left[n^{\lambda} \log n\right]$ gaps of lenght $n^{1-\lambda}$ )

Since the Hausdorff dimension of a Cantor set

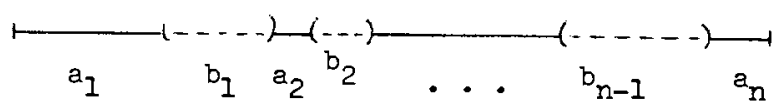

normalized such that $\sum_{k=1}^{n} a_{k}+\sum_{j=1}^{n-1} b_{j}=1$, is the only $\mu \in(0,1)$ such that $\sum_{k=1}^{n} a_{k}^{\mu}=1$ (see [PT 2]), we have $H D\left(K_{n}^{\lambda}\right)=\mu$, where $\mu$ satisfies

$$
\begin{aligned}
& 2\left(\frac{n}{2 n+2\left(\left[n^{\lambda} \log n\right]-1\right)+n^{1-\lambda}\left[n^{\lambda} \log n\right]}\right)^{\mu} \\
& \quad+\left(\left[n^{\lambda} \log n\right]-1\right)\left(\frac{2}{2 n+2\left(\left[n^{\lambda} \log \right]-1\right)+n^{1-\lambda}\left[n^{\lambda} \log n\right]}\right)^{\mu}=1
\end{aligned}
$$

If we denote by $g_{\lambda}(n, \mu)$ the left side of this equation, and observe that $2 n+2\left(\left[n^{\lambda} \log n\right]-1\right)+n^{1-\lambda}\left[n^{\lambda} \log n\right]$ is of the order of $n \log n$, we conclude that $\mu>\lambda \Rightarrow \lim _{n \rightarrow \infty} g_{\lambda}(n, \mu)=0$, and $\mu<\lambda \Rightarrow \lim _{n \rightarrow \infty} g_{\lambda}(\eta, \mu)=+\infty$. Thus, $\lim _{n \rightarrow \infty} H D\left(K_{n}^{\lambda}\right)=\lambda$, and then there exists $n_{0} \in \mathbf{N}$ such that $H D\left(K_{n_{0}}^{a}\right)<h_{1}$ and $H D\left(K_{n_{0}}^{b}\right)<h_{2}$. We are
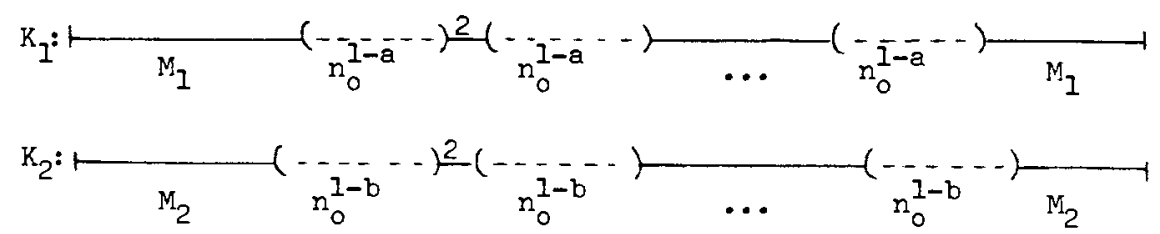

where $M_{1} \geq n_{0}$ and $M_{2} \geq n_{0}$ are choosen such that $H D\left(K_{1}\right)=h_{1}$ and $H D\left(K_{2}\right)=h_{2}(H D(K)$ depends continuously on $K)$. 
$\left(K_{1}, K_{2}\right)$ satisfies the GTT: We have $\frac{M_{1}}{n_{0}^{1-a}} \cdot \frac{2}{n_{0}^{1-b}} \geq \frac{2 \cdot n_{0}}{n_{0}^{2-a-b}} \geq 2>1$ and $\frac{M_{2}}{n_{0}^{1-b}} \cdot \frac{2}{n_{0}^{1-a}} \geq 2>1$. Thus, studying intersections of pairs $\left(P_{1}, \lambda P_{2}+t\right)$, where $P_{i}$ is the Markov domain of $K_{i}$ let us suppose, for instance, that the right interval $I_{1}$ of $P_{1}$ (but not all the $P_{n}$ ) is contained in a gap $U=(a, b)$ of $\lambda P_{2}+t$. Let $\tilde{U}$ be the gap of $P_{1}$ that contains $a$ and $I_{2}=[c, a]$ the interval of $\lambda P_{2}+t$ at the right of $(a, b)$. We have $\frac{\left|I_{1}\right|}{|\tilde{U}|} \cdot \frac{\left|I_{2}\right|}{|U|} \geq \frac{M_{1}}{n_{0}^{1-b}} \cdot \frac{2}{n_{0} 0^{1-b}}>1$, and $\left|I_{1}\right|<|U| \Rightarrow\left|I_{2}\right|>|\tilde{U}| \Rightarrow P_{1}$ intersects $\lambda P_{2}+t$. The other possible positions of $\left(P_{1}, \lambda P_{2}+t\right)$ have similar analysis. By the arguments above it is clear that $\left(K_{1}, K_{2}\right)$ satisfies the GTT stably.

In the example IV.1, we have constructed a Cantor set $\bar{K} \subset K$ with $\tau(\bar{K})>\tau(K)$ by simply forgetting some intervals of $K$, and considering the Cantor set defined by the same function restricted to the remaining intervals. This can be done even if some of the forgotten intervals are extremal, provided that the Markov partition of $K$ has more than two intervals. In this case the description of $\bar{K}$ is slightly more complicated: Indeed, let $K$ be a Cantor set with Markov partitions $\left\{K_{1}, K_{2}, \ldots, K_{n}\right\}$ defined by an expanding function $\psi$, such that $\left.\psi\right|_{K_{1}}$ is increasing and surjective. If $\left.\psi\right|_{K_{n-1}}$ is increasing and surjective, then $\left.\psi\right|_{K_{n-1}}$ has an unique fixed point $b$. If $\left.\psi\right|_{K_{n-1}}$ is decreasing and surjective, we take $b$ equal to the right extreme of $K_{n-1}$. Then we have a dynamically defined Cantor set $\bar{K}$ contained in $K$ with Markov domain $\left\{\bar{K}_{1}, \bar{K}_{2}, \ldots \bar{K}_{n-1}\right\}$ where $\bar{K}_{i}=\psi^{-1}([0, b]) \cap K_{i}, i=1,2, \ldots, n-1$, defined by the (the restriction of) $\psi$.

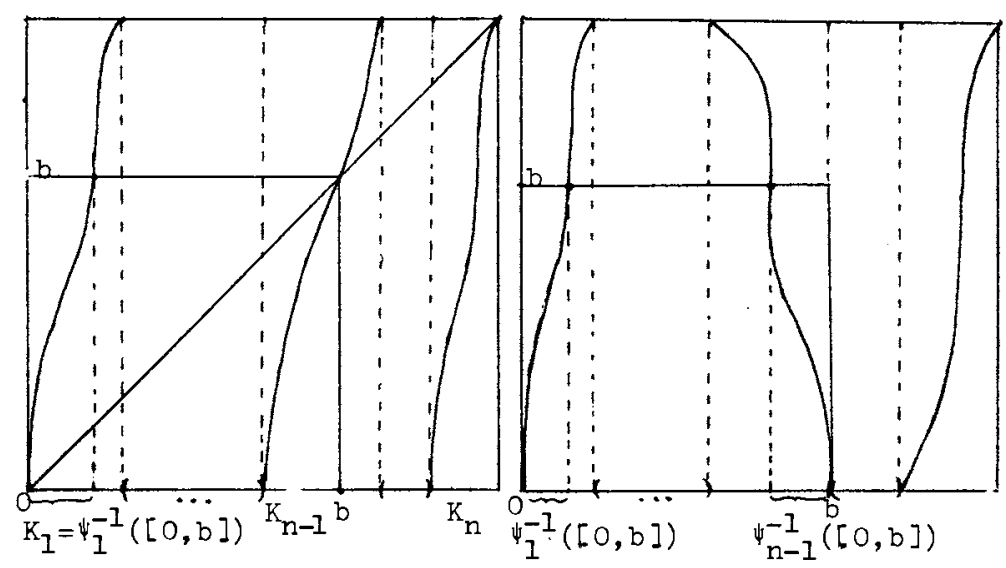

This Cantor set does not depend on $K_{n}$ or on $\left.\psi\right|_{K_{n}}$. 
As an example, if

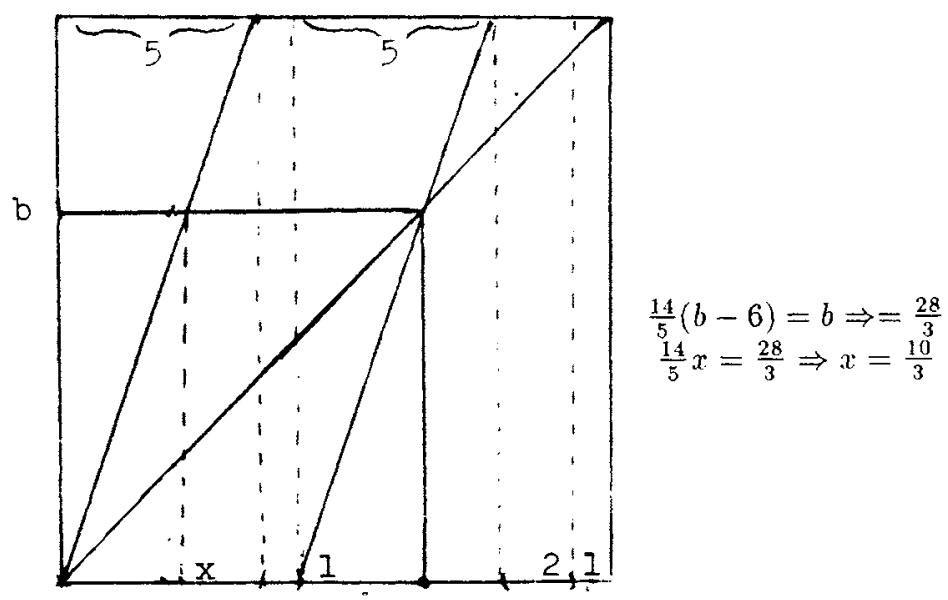

If $\tilde{K}=\longmapsto 1, \ldots 1 \ldots+1$

then clearly the pair $(K, \tilde{K})$ does not satisfy the GTT, as showed in the picture below:

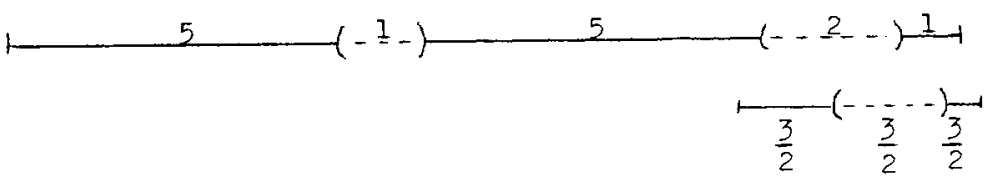

However, $\tau(\bar{K}) \tau(\tilde{K})=\frac{5}{4} 1=\frac{5}{4}>1$, therefore $(\tilde{K}, \bar{K}+3)$ has extremal stable intersection, and so does $(\tilde{K}, K+3)$, since $\bar{K}$ depends continuously on $K$.

On the other hand, $(K, \tilde{K}+14)$ does not have extremal stable intersection, by Theorem IV.1, and so the existence of extremal stable intersection depends on the order of the pair of Cantor sets.

Let us make a definition: Given compact sets $C_{1}$ and $C_{2}$ with convex hulls $[a, b]$ and $[c, d]$, respectively, we say that $C_{1} \rightarrow C_{2}: \Leftrightarrow(a<c, b<d$ and $c<b$ ).

Theorem IV.1. - Let $(K, \bar{K})$ be a pair of dynamically defined Cantor sets whose Markov partitions are $P=\left\{K_{1}, K_{2}, \ldots, K_{n}\right\}$ and $\bar{P}=$ $\left\{\bar{K}_{1}, \bar{K}_{2}, \ldots, \bar{K}_{2}\right\}$ respectively, such that the right extreme of $K$ coincides 
with the left one of $\bar{K}$. Suppose that $\left.\psi\right|_{K_{n}}$ and $\left.\bar{\psi}^{\prime}\right|_{\bar{K}_{1}}$ are affine, increasing and surjective, and that $\log \left(\left.\psi^{\prime}\right|_{K_{n}}\right) / \log \left(\left.\bar{\psi}^{\prime}\right|_{\bar{K}_{1}}\right) \notin \mathrm{Q}$, where $\psi$ and $\bar{\psi}$ are the functions that define $K$ and $\bar{K}$. Then: $\bar{K}-K \not \supset[0, \delta], \forall \delta>0 \Leftrightarrow$ $\exists \lambda>0, t \in \mathbf{R}$ s.t. $(\lambda K+t) \rightarrow \bar{K}$ and $(\lambda K+t) \cap \bar{K}=\emptyset$.

\section{Remarks.}

- This last condition is equivalent to the existence of a straight line $\ell$ which crosses only the upper and the right sides of $K_{0} \times \bar{K}_{0}$ and does not intersect some step $P_{i} \times \bar{P}_{i}$ of the construction of $K \times \bar{K}$, where $P_{i}=\psi^{1-i}(P), \bar{P}_{i}=\bar{\psi}^{1-i}(\bar{P})$, and $K_{0}$ and $\bar{K}_{0}$ are respectively the convex hulls of $K$ and $\bar{K}$.

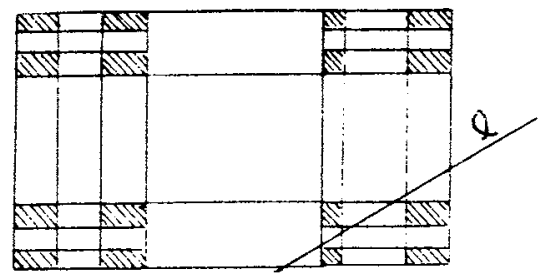

- We can supress the condition $\log \left(\left.\psi^{\prime}\right|_{K_{n}}\right) / \log \left(\psi^{\prime} / \bar{K}_{1}\right) \notin Q$ if we change the last condition by $\exists t \in \mathbf{R}$ such that $(K+t) \rightarrow \bar{K}$ and $(K+t) \cap \bar{K}=\emptyset$.

- If $\left.\psi\right|_{K_{n}}$ and $\left.\psi\right|_{\bar{K}_{1}}$ are increasing and surjective but not affine, then the results are also valid, provided that we test the hypothesis for the linearized of $K$ and $\bar{K}$ (for which the analogs of $\left.\psi\right|_{K_{n}}$ and $\left.\bar{\psi}\right|_{\bar{K}_{1}}$ are affine).

Proof. - Let $\lambda_{1}=\left.\psi^{\prime}\right|_{K_{n}}$ and $\log \lambda_{1} / \log \lambda_{2} \notin Q$, there are, for each $\delta>0$, an infinity of pairs of natural numbers $(m, n)$ such that $\left|\lambda_{2}^{m} / \lambda_{2}^{n}-\lambda\right|<\delta$. Since $t \notin \bar{K}-\lambda K$, there is $\delta>0$ such that $t^{\prime} \notin \bar{K}-\lambda^{\prime} K \quad \forall \lambda^{\prime}, t^{\prime}$ with $\left|t-t^{\prime}\right|<\delta$, and $\left|\lambda-\lambda^{\prime}\right|<\delta \Rightarrow t \notin$ $\bar{K}-\left(\lambda_{2}^{n} / \lambda_{1}^{m}\right) K \Rightarrow \lambda_{1}^{-n} t \notin \lambda_{2}^{-n} \bar{K}-\lambda_{1}^{-m} K$, with $\left(\lambda_{1}^{m} K+\lambda_{2}^{-n} t\right) \rightarrow \lambda_{2}^{-n} \bar{K}$, and, since $\bar{K}_{0} \cap\left(K_{0}+\lambda_{2}^{-n} t\right)=\left(\lambda_{1}^{-m} K_{0}+\lambda_{2}^{-n} t\right) \cap\left(\lambda_{2}^{-n} K_{0}\right), \lambda_{2}^{n} t \notin \bar{K}-K$. Therefore, $\bar{K}-K \not \supset[0, \varepsilon], \forall \varepsilon>0$. The other implication of this theorem is clear.

Remark 1. - The above demonstration also shows that, as in Theorem I.2, in the hypothesis of Theorem IV.1 $\left(K_{2}-K_{1}\right)^{c}$ has positive density at 0 . We shall adapt this result in section $\mathrm{V}$, to show that in the case of homoclinic bifurcations, if $K=K^{s}$ and $\bar{K}=K^{u}$ satisfy the hypothesis of the Theorem IV.1, then we shall have $\varphi_{\mu}$ persistently hyperbolic for a set of positive values of $\mu$ with positive density at $\mu=0$. 
Remark 2. - If $K_{1}$ and $K_{2}$ are affine Cantor sets satisfying the hypothesis of Theorem IV.1, if there are $\lambda>0$ and $t \in \mathbf{R}$ such that $K_{1} \rightarrow\left(\lambda K_{2}+t\right)$ and $K_{1} \cap\left(\lambda K_{2}+t\right)=\emptyset$ then, by this theorem, $\left(K_{1}, K_{2}\right)$ will not have extremal stable intersection, since (Perhaps by changing slightly the pair $\left(K_{1}, K_{2}\right)$ to satisfy the hypothesis about $\lambda_{1}$ and $\left.\lambda_{2}\right)$ its arithmetic difference will not contain $[0, \varepsilon], \forall \varepsilon>0$. Consequently, if

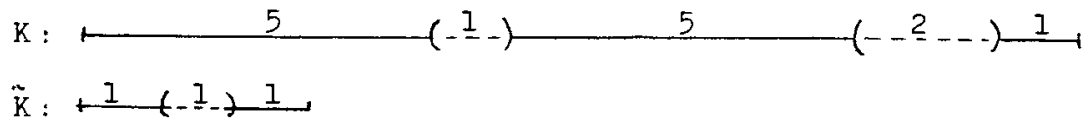

then $(K, \bar{K}+8)$ will not have extremal stable intersection, as we stated before. The statements in of the examples IV.4 and IV.5 also follow.

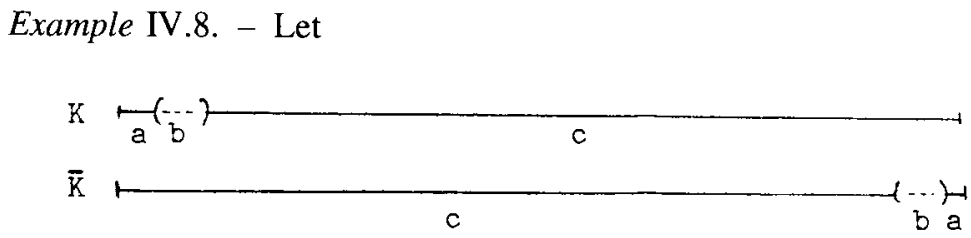

where $a+b+c=1$. We remember that $H D(K)=H D(\bar{K})=\lambda$, where $\lambda$ is the only real number such that $a^{\lambda}+c^{\lambda}=1$. Therefore, even if we chose $b$ small, we can choose $a$ so small that $H D(K)=H D(\bar{K})<\frac{1}{2}$. In this case, since $H D(K)+H D(\bar{K})<1$, we have $m(K-\bar{K})=0$, and so $(K, \bar{K}+1)$ will not have extremal stable intersection, and $K-\bar{K}$ will not contain any interval. However, to apply Theorem IV.1, we must consider very advanced steps of the construction of $K \times \bar{K}$, even in this case, which is combinatorially very simple.

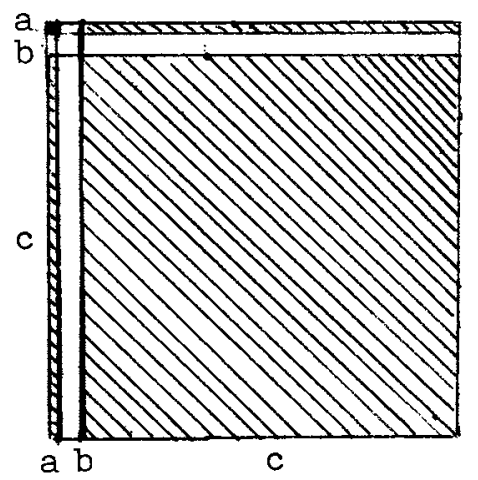

Annales de l'Institut Henri Poincaré - Analyse non linéaire 
Example IV.9. - Let

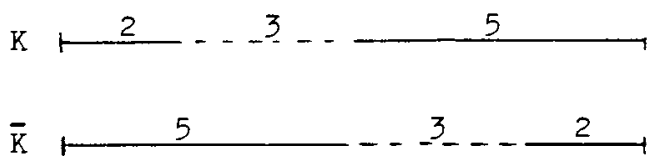

The first step of the construction of $K \times \bar{K}$ does not assure that $(K, \bar{K}+10)$ does not have a stable intersection, but the second step does:
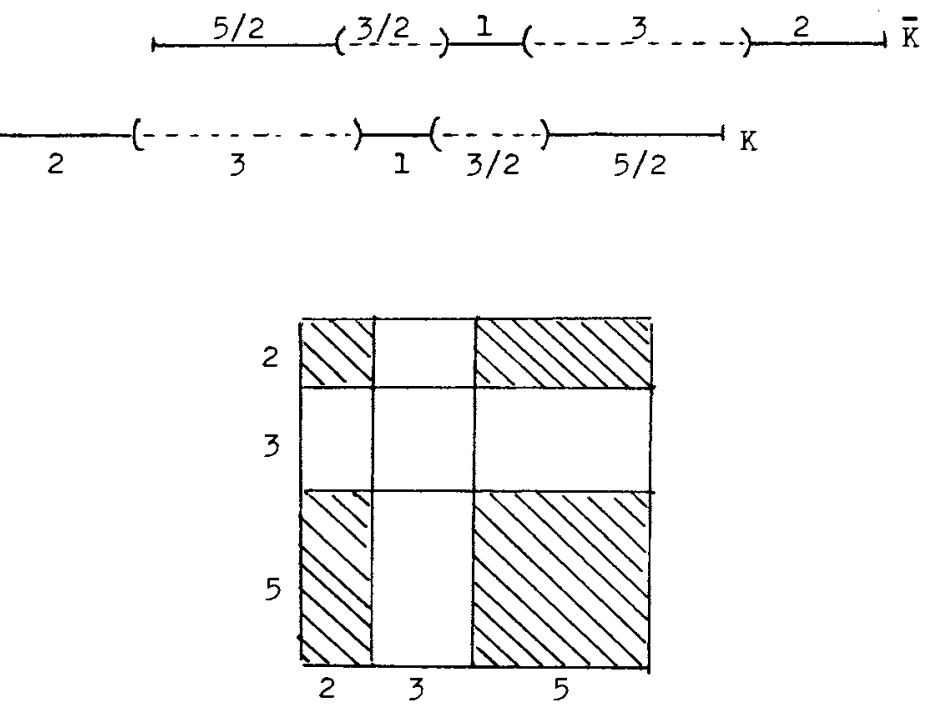

\section{INTERSECTIONS OF CANTOR SETS AND HYPERBOLICITY}

In [PT1], Palis and Takens proved the following theorem:

Let $\left\{\varphi_{\mu} \mid \mu \in \mathbf{R}\right\}$ be a family of diffeomorphisms of $M^{2}$ with an homoclinic $\Omega$-explosion at $\mu=0$. Suppose that $H D\left(K^{s}\right)+H D\left(K^{u}\right)<1$. Then

$$
\lim _{\delta \rightarrow 0} \frac{m\left(\left\{\mu \in[0, \delta] \mid \varphi_{\mu} \text { is persistently hyperbolic }\right\}\right)}{\delta}=1
$$

where $m$ denotes the Lebesgue measure.

Vol. 13, $\mathrm{n}^{\circ}$ 6-1996. 
In [PY], Palis and Yoccoz proved that if $H D\left(K^{s}\right)+H D\left(K^{u}\right)>1$ then generically

$$
\limsup _{\delta \rightarrow 0} \frac{m\left(\left\{\mu \in[0, \delta] \mid \varphi_{\mu} \text { is not persistently hyperbolic }\right\}\right)}{\delta}>0,
$$

because we have new tangencies between $W^{s}(\Lambda)$ and $W^{u}(\Lambda)$ with positive upper density at $\mu=0$, where $\Lambda$ is the basic set of $\varphi_{0}$ associated to the homoclinic tangency.

Here we shall prove that if $K^{s}$ and $K^{u}$ satisfy the hypothesis of Theorem IV.1, then we have hyperbolicity with positive density at $\mu=0$, even if $H D(\Lambda)>1$. The proof of this result will be a combination of the arguments in [PT1] and Theorems I.2 and IV.1. We shall also give an example of an open set of families $\left(\varphi_{\mu}\right)$ where the phenomena of hyperbolicity and of new tangencies between $W^{s}(\Lambda)$ and $W^{u}(\Lambda)$ have both positive density at $\mu=0$.

THEOREM V.1. - Let $\left(\varphi_{\mu}\right)$ be a family of diffeomorphisms of $M^{2}$ that presents a homoclinic $\Omega$-explosion at $\mu=0$ associated to a fixed saddle point $p$ that belongs to a basic set $\Lambda$, and let $\left(\tilde{K}_{1}\right)_{0}$ and $\left(\tilde{K}_{2}\right)_{0}$ be as in Theorem I.2. Suppose further that there is $t \in \mathbf{R}$ such that $\left(\left(\tilde{K}_{1}\right)_{0}+t\right) \rightarrow\left(\tilde{K}_{2}\right)_{0}$ and $\left(\left(\tilde{K}_{1}\right)_{0}+t\right) \cap\left(\tilde{K}_{2}\right)_{0}=\emptyset$.

Then

$$
\liminf _{\delta \rightarrow 0} \frac{m\left(\left\{\mu \in[0, \delta] \mid \varphi_{\mu} \text { is persistently hyperbolic }\right\}\right)}{\delta}>0 .
$$

Remark. - In the hypothesis of this theorem, as in the hypothesis of Theorem I.2, we suppose that $\left(\tilde{K}_{1}\right)_{0} \subset \mathbf{R}_{-},\left(\tilde{K}_{2}\right)_{0} \subset \mathbf{R}_{+}$and $0 \in\left(\tilde{K}_{1}\right)_{0} \cap\left(\tilde{K}_{2}\right)_{0}$.

Proof. - Let $\mathcal{O}$ the orbit of homoclinic tangency, $q^{s} \in \mathcal{O} \cap W_{\text {loc }}^{s}(p)$ and $q^{u} \in \mathcal{O} \cap W_{\text {loc }}^{u}(p)$. Let $q_{\mu}^{s} \in W_{\text {loc }}^{s, \mu}\left(p_{\mu}\right)$ and $q_{\mu}^{u} \in W_{\text {loc }}^{u, \mu}\left(p_{\mu}\right)$ depending continuously on $\mu$ such that $q_{0}^{s}=q^{s}$ and $q_{0}^{u}=q^{u}$ and $q_{0}^{u}=q^{u}$, and let $\mathcal{O}_{\mu}^{s}$ and $\mathcal{O}_{\mu}^{u}$ their $\varphi_{\mu}$-orbits. Let $A(\mu)=K_{\mu}^{s} \cup \mathcal{O}_{\mu}^{s}$ and $B(\mu)=K_{\mu}^{u} \cup \mathcal{O}_{\mu}^{u}$, where $K_{\mu}^{s}=W_{\text {loc }}^{s, \mu}\left(p_{\mu}\right) \cap \Lambda_{\mu}$ and $K_{\mu}^{u}=W_{\text {loc }}^{u, \mu}\left(p_{\mu}\right) \cap \Lambda_{\mu}$. ( $\Lambda_{\mu}$ is the continuation of $\Lambda$ ). Choose parametrizations for $W_{\mathrm{loc}}^{s, \mu}\left(p_{\mu}\right)$ and $W_{\mathrm{loc}}^{u, \mu}\left(p_{\mu}\right)$ (for which $\left.\varphi_{\mu}\right|_{W_{10 c}^{s, \mu}}\left(p_{\mu}\right)$ and $\left.\varphi_{\mu}\right|_{W_{\text {loc }}^{s, \mu}}\left(p_{\mu}\right)$ are linear maps) in such a way that $K_{\mu}^{s}$ and $K_{\mu}^{u}$ coincides with $\left(\tilde{K}_{1}\right)_{\mu}$ and $\left(\tilde{K}_{2}\right)_{\mu}$, and that $A(\mu)$ and $B(\mu)$ are scaled sets, with scaling factors $\lambda_{1}(\mu)$ and $\lambda_{2}(\mu)$, respectively, where 
$\lambda_{1}(\mu)$ and $\lambda_{2}(\mu)$ are the eigenvalues of $\varphi_{\mu}$. For a set $A \subset \mathbf{R}$ we define ${ }_{\delta} A=\left\{x \in \mathbf{R} \mid \exists x^{\prime} \in A\right.$ such that $\left.\left|x-x^{\prime}\right| \leq \delta\left|x^{\prime}\right|\right\}$.

Let ${ }_{\varepsilon} \tilde{A}^{K \cdot \mu}\left(\mu^{\prime}\right)=\pi_{s, \mu^{\prime}}^{-1}\left({ }_{\varepsilon} A\left(\mu^{\prime}\right) \cap[-K \mu, K \mu]\right)$, and ${ }_{\varepsilon} \tilde{B}^{K \cdot \mu}=$ $\pi_{u, \mu^{\prime}}^{-1}\left({ }_{\varepsilon} B\left(\mu^{\prime}\right) \cap[-K \mu, K \mu]\right)$ where $\pi_{s, \mu^{\prime}}$ and $\pi_{u, \mu^{\prime}}$ are the projection associated to the stable and unstable foliations of $\varphi_{\mu^{\prime}}$ over the line of tangencies $\ell_{\mu}$. Let $C_{\mu, \varepsilon}=\left\{\mu^{\prime} \in(0, \mu) \mid\right.$ the distance between ${ }_{\varepsilon} \tilde{B}^{K \mu}\left(\mu^{\prime}\right)$ and ${ }_{\varepsilon} \tilde{A}^{K \mu}\left(\mu^{\prime}\right)$ is greater or equal to $\left.\varepsilon \mu\right\}$. If we could prove that $\liminf _{\mu \rightarrow 0} \frac{m\left(C_{\mu, \varepsilon}\right)}{\mu}>0$ for some $\varepsilon>0$ then the result would follow exactly as in [PT1].

Let ${ }_{\varepsilon} \tilde{\mathcal{O}}_{\mu^{\prime}}^{s, K_{\mu}}=\pi_{s, \mu^{\prime}}^{-1}\left({ }_{\varepsilon} \mathcal{O}_{\mu^{\prime}}^{s}\left(\mu^{\prime}\right) \cap[-K \mu, K \mu]\right)$, and ${ }_{\varepsilon} \tilde{\mathcal{O}}_{\mu^{\prime}}^{u, K \cdot \mu}=$ $\left.\pi_{u, \mu^{\prime}}^{-1}\left({ }_{\varepsilon} \mathcal{O}_{\mu^{\prime}}^{u}\right) \cap[-K \mu, K \mu]\right)$ then $C_{\mu, \varepsilon}^{\prime}=\left\{\mu^{\prime} \in(0, \mu)\right.$ $d\left({ }_{\varepsilon} A^{K \mu}\left(\mu^{\prime}\right),{ }_{\varepsilon} \mathcal{O}_{\mu^{\prime}}^{u, K \mu}\right) \geq \varepsilon \mu$ and $\left.d\left({ }_{\varepsilon} \mathcal{O}_{\mu^{\prime}}^{s, k \mu},{ }_{\varepsilon} B^{K \mu}\left(\mu^{\prime}\right)\right) \geq \varepsilon \mu\right\}$ is such that given $\delta>0$ there is $\varepsilon>0$ for which we have $\liminf _{\mu \rightarrow 0} \frac{m\left(C_{\mu, \varepsilon}^{\prime}\right)}{\mu}>1-\delta$, by the arguments of [PT1], since $\mathcal{O}_{\mu}^{s}$ and $\mathcal{O}_{\mu}^{u}$ are countable, and thus have Hausdorff dimension zero.

Let $\tilde{K}_{\mu^{\prime}}^{u, \mu}=\pi_{u, \mu^{\prime}}^{-1}\left({ }_{\varepsilon} \tilde{K}_{\mu^{\prime}}^{u} \cap[-K \mu, K \mu]\right)$, and $C_{\mu^{\prime}, \varepsilon}^{\prime}=\{\mu \in(0, \mu) \mid$ $\left.d\left({ }_{\varepsilon} \tilde{K}_{\mu^{\prime}{ }_{\varepsilon}}^{u, \mu} \tilde{K}_{\mu^{\prime}}^{s, \mu}\right) \geq \varepsilon \mu\right\}$. Then we can prove, by arguments similar to the arguments of Theorem I.2, that if $\varepsilon>0$ is sufficiently small then $\liminf _{\mu \rightarrow 0}$ $\frac{m\left(C_{\mu, \varepsilon}^{\prime}{ }^{\prime}\right)}{\mu}>0$ (the essence of this argument is that "non-intersections" are always stable). Thus, if $\varepsilon>0$ is sufficienlty small then is $\liminf _{\mu \rightarrow 0}$ $\frac{m\left(C_{\mu, \varepsilon}^{\prime}{ }^{\prime}\right)}{\mu}>0$, and the theorem is proved.

Remark. - If $\log \left(\lambda_{1}(0)\right) / \log \left(\lambda_{2}(0)\right) \notin \mathbf{Q}$, it is enough to suppose that there are $\lambda>0, t \in \mathbf{R}$ such that $\left(\lambda\left(\tilde{K}_{1}\right)_{0}+t\right) \rightarrow\left(\tilde{K}_{2}\right)_{0}$ and $\left(\lambda\left(\tilde{K}_{1}\right)_{0}+t\right) \cap\left(\tilde{K}_{1}\right)_{0}=\emptyset$.

Example. - Suppose that $K_{0}^{s}$ and $K_{0}^{u}$ are affine Cantor sets as in the pictures $\mathrm{B}$ and $\mathrm{C}$ below, associated to a horseshoe as in pinture $\mathrm{A}$, which is combinatorially equivalent to the square of the usual horseshoe.

Let us suppose that $\left(\varphi_{\mu}\right)$ presents a homoclinic $\Omega$-explosion at $\mu=0$ associated to the fixed point $p$ in the left lower extreme of $\Lambda$. Then, since the hypothesis of the Theorem V.1 are satisfacted, we shall have hyperbolicity with positive density at $\mu=0$.

On the other hand, as the picture $D$ shows, $K_{0}^{s}$ contains an affine Cantor set with thickness $17 / 10$ (we "forget" the right interval). Similarly, we can show that $K_{0}^{u}$ contains an affine Cantor set with thickness greater than $1 \Rightarrow$ there is a $t \in \mathbf{R}$ such that $\left(K_{0}^{s}+t\right)$ intersects $K_{0}^{u}$ stably, and so, according to Theorem I.2, there will be tangencies between $W^{s}\left(\Lambda_{\mu}\right)$ and 


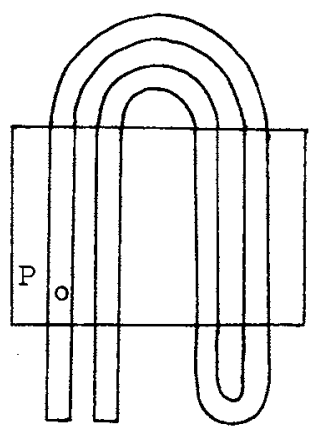

A

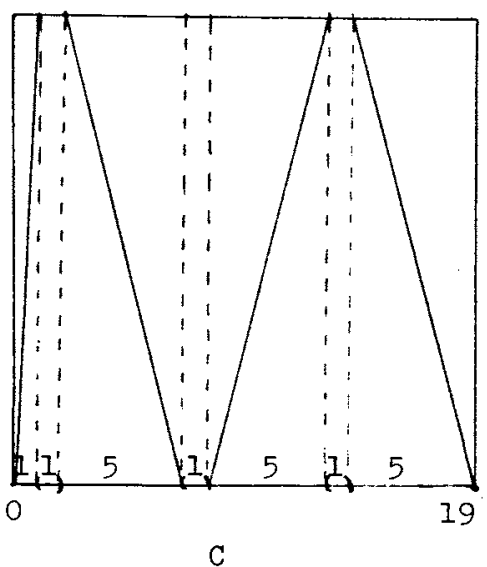

C

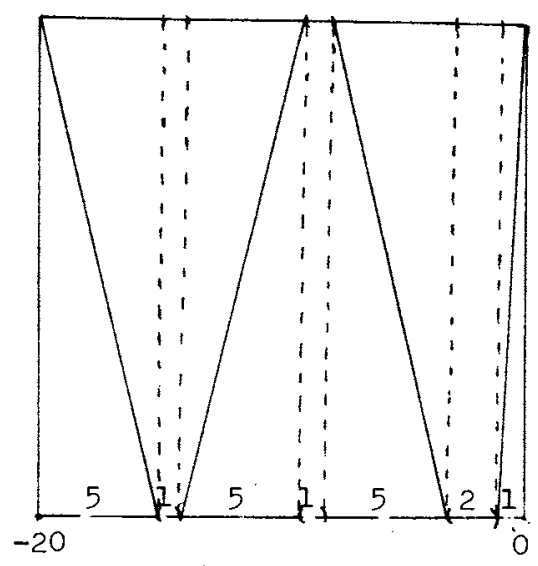

$\mathrm{B}$

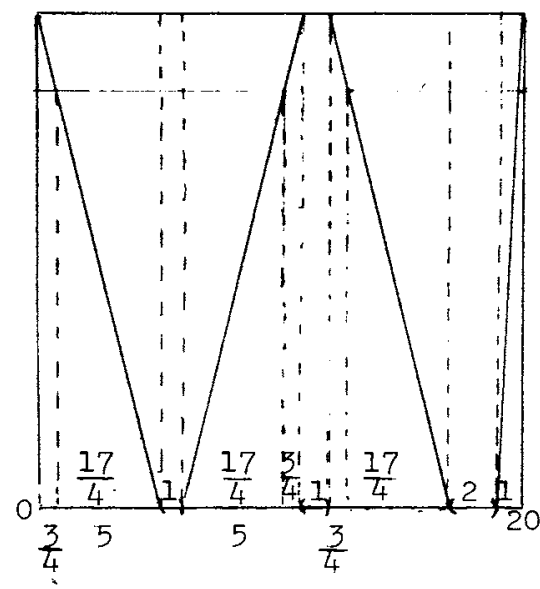

D

$W^{u}\left(\Lambda_{\mu}\right)$ with positive density at $\mu=0$. The same phenomena occur in a neighbourhood of $\left(\varphi_{\mu}\right)$, since the hypothesis are open.

\section{ACKNOWLEDGEMENTS}

I would like to thank J. Palis, for posing to me the problem and for his constant support. Also to J. C. Yoccoz, M. Viana, P. Mendes, S. Plaza and A. Sannami for stimulating and helpful conversations. I want also to stress the fine mathematical ambiance of IMPA, where this work was done. 


\section{REFERENCES}

[BPV] R. BAmón, S. Plaza and J. VERA, On Central Cantor Sets with self-arithmetic difference of positive Lebesgue measure, to appear in J. London Math. Soc.

[H] M. HALL, On the sum and product of continued fractions, Annals of Math., Vol. 48, 1947, pp. 966-993.

[MO] P. MENDES and F. Oliveira, On the topological structure of the arithmetic sum of two Cantor sets, Nonlinearity, Vol. 7, 1994, pp. 329-343.

[N1] S. Newhouse, Non density of Axiom $A(a)$ on $S^{2}$, Proc. A.M.S. Symp. Pure Math., Vol. 14, 1970, pp. 191-202.

[N2] S. Newhouse, Diffeomorphisms with infinitely many sinks, Topology, Vol. 13, 1974, pp. $9-18$

[N3] S. NEwhouse, The abundance of wild hyperbolic sets and nonsmooth stable sets for diffeomorphisms, Publ. Math. IHES, Vol. 50, 1979, pp. 101-151.

[P] J. PALIS, Homoclinic bifurcations, sensitive chaotic dynamics and strange attractors, Dynamical Syst. and Related Topics, World Scientific, 1991, pp. 466-473.

[PT] J. PALIS and F. TAKENS, Cycles and measure of bifurcation sets for two-dimensional diffeomorphisms, Invent. Math., Vol. 82, 1985, pp. 379-442.

[PT1] J. PALIS and F. TAKENS, Hyperbolicity and the creation of homoclinic orbits, Annals of Math., Vol. 125, 1987, pp. 337-374.

[PT2] J. PALIS and F. TAKENS, Hyperbolicity and sensitive chaotic dynamics at homoclinic bifurcations: fractal dimensions and infinitely many attractors, Cambridge Univ. Press, 1992.

[PY] J. PALIS and J. C. Yoccoz, Homoclinic Tangencies for Hyperbolic sets of large Hausdorff Dimension Bifurcations, Acta Mathematica, Vol. 172, 1994, pp. 91-136.

[S] A. Sannami, An example of a regular Cantor set whose difference set is a Cantor set with positive measure, Hokkaido Math. Journal, Vol. XXI (1), 1992, pp. 7-23

(Manuscript received October 25, 1994.) 